\title{
STRANGE ATTRACTORS OF UNIFORM FLOWS ${ }^{1}$
}

\author{
BY
}

ITTAI KAN

\begin{abstract}
Consider orbitally stable attractors of those flows on the open solid torus $D^{2} \times S^{1}$ which have uniform velocity in the $S^{1}$ direction (uniform flows). It is found that any such attractor is the frontier of a strictly nested sequence of positively invariant open solid tori. Necessary and sufficient conditions related to these tori are derived for an arbitrary set to be an orbitally stable attractor. When the cross-section of an orbitally stable attractor is a Cantor set, the first return map is found to be conjugate to an irrational rotation on a certain compact abelian group. New examples are constructed of orbitally stable attractors of uniform $C^{\infty}$ flows whose cross-sections have uncountably many components (one of these attractors has positive 3-dimensional Lebesgue measure).
\end{abstract}

\section{List of symbols.}

$T \quad$ Standard open solid torus.

$A_{\theta} \quad$ Cross-section of $A$ with $\theta$ held constant. ${ }^{2}$

$\bar{A} \quad$ Closure of $A$.

INT(A) Interior of $A$.

$C A \quad$ Complement of $A$.

$\operatorname{Fr}(A) \quad$ Frontier of $A=\bar{A} \cap \overline{C A}$.

$N(A, \varepsilon) \quad$ Open $\varepsilon$-neighborhood of $A$.

$d(\cdot, \cdot) \quad$ Product of the euclidean metric on $D^{2}$ and the arc-length metric on $S^{\prime}$. Also used to denote the usual distance between sets.

$C^{k} \quad k$ times continuously boundedly differentiable.

@(A) Number of components of $A$.

\#(TA) Winding number of $A$.

$\mathscr{B}(A) \quad$ Basin of attraction of $A$.

$\omega(x) \quad \omega$-limit set of $x$.

$D \quad$ Derivative operator.

$\|\cdot\|_{n} \quad$ Supremum norm on the $n$th directional derivative.

$\mathrm{HD}(A) \quad$ Hausdorff dimension of $A$.

Received by the editors November 16, 1984.

1980 Mathematics Subject Classification. Primary 58F12.

Key words and phrases. Attractors, strange attractors, Hausdorff dimension.

${ }^{1}$ Substantially the same as Ph.D. thesis with same title.

${ }^{2}$ Unless otherwise stated $\theta=0$. When we refer to the topology of $E_{\theta}$ (closure, interior, frontier, complement, neighborhood, etc.) we mean relative to the plane, and omit the $\theta$ term from our coordinates. 
$\mathrm{HD}_{l}(A, x)$ Local Hausdorff dimension of $A$ at $x$.

$\mathscr{M} \quad$ Normalized Lebesgue 3-measure.

$\omega$

Cardinality of the integers (we use cardinals as ordinals and viceversa in

the usual way).

$I \cdot I \quad$ Greatest integer part.

Introduction. Flows of time-periodic vector fields on the planar disk are used to model a multitude of physical phenomena. We make the usual transformation and consider these flows as uniform flows on the standard open solid torus $T$.

Definition 1. A uniform flow $\varphi_{t}$ is a one-parameter group of automorphisms of the standard open solid torus

$$
T=\{(z, \theta)|z \in \mathbf{C},| z \mid<1, \theta \in \mathbf{R} \bmod (2 \pi)\},
$$

with $\varphi_{s} \circ \varphi_{t}=\varphi_{s+t}$ for all $s, t \in \mathbf{R}$, and with $\varphi_{t}(\cdot, \theta)=(\cdot,(\theta+t) \bmod (2 \pi))$.

An attractor, when one exists, is useful in describing the long term behavior of the trajectories it attracts. Unfortunately, although simple attractors of uniform flows such as the limit cycle or the 2-torus, and more complicated attractors such as a uniform version of the Plykin attractor [Plykin, 1974; Newhouse, 1980], are well understood, there are uniform flows such as the flow of the Duffing equation [Duffing, 1918; Guckenheimer and Holmes, 1983], the study of which has produced numerical evidence for the existence of a complicated attractor whose precise nature is unclear, and whose existence has neither been proven nor disproven. Furthermore, given an arbitrary set $A \subset T$ there is no known general method for determining whether or not $A$ can be imbedded as an attractor of some uniform $C^{0}$ flow, and if $A$ can be imbedded in a uniform $C^{0}$ flow $\varphi_{t}$, then what the geometry of $A$ implies about the dynamics of $\varphi_{t}$ is not well understood.

In this paper we consider orbitally stable (Definition 3) attractors (Definition 2) of uniform $C^{0}$ flows. We find that the geometrical properties of such attractors have important constraints which are closely related to dynamical features. We also relate the dynamics of the first return map on the cross-sections of certain orbitally stable attractors to the degree of differentiability of the uniform flow in which the map is imbedded. In addition, we construct uniform $C^{\infty}$ flows yielding new examples of orbitally stable attractors which have a variety of interesting properties. Finally, we investigate the Hausdorff dimension of orbitally stable attractors of uniform Lipschitz flows.

DEFINITION 2. A compact nonempty set $A \subset T$ is called an attractor of a uniform flow $\varphi_{t}$ if $A$ is the $\omega$-limit set of almost every point in a deleted neighborhood of $A$.

There are a variety of definitions of attractor in the literature. By our definition the set $T$ itself cannot be an attractor, and any attractor must be connected and invariant. The basin of attraction of an attractor $A$ is the closure of the set of points whose $\omega$-limit set is $A$.

Definition 3. An attractor $A \subset T$ of a uniform flow $\varphi_{t}$ is called orbitally stable if any neighborhood $U$ of $A$ contains a subneighborhood $V$ of $A$ such that $\varphi_{t}(V) \subset U$ for all $t>0$. 
We now briefly describe the results of this paper. We find it useful to partition the geometries of certain sets, which might be imbeddable as attractors, according to the following scheme.

Definition 4. If there exists a uniform $C^{0}$ flow under which the compact set $A \subset T$ is invariant, then the class of $A$ is an ordered triple $\mathscr{C}(\alpha, \beta, \gamma)$, where:

(1) $\alpha$ is the number of components in a cross-section of $A$.

(2) $\beta$ is 0 or 1 , depending on whether the components of a cross-section of $A$ are all singleton points or are not all singleton points, respectively.

(3) $\gamma$ is the number of components in the complement of $A$.

For example, when $A$ is the 2-torus we have class $(A)=\mathscr{C}(1,1,2)$.

The following six collections of classes will be of particular interest.

Definition 5. A class is called permissible if it lies in one of these six collections.

(i) $\left\{\mathscr{C}(N, 0,1) \mid N \in \mathbf{Z}^{+}\right\}$,

(ii) $\left\{\mathscr{C}(N, 1,1) \mid N \in \mathbf{Z}^{+}\right\}$,

(iii) $\left\{\mathscr{C}(N, 1, M) \mid N, M \in \mathbf{Z}^{+}, M \geqslant 2\right\}$,

(iv) $\left\{\mathscr{C}(N, 1, \omega) \mid N \in \mathbf{Z}^{+}\right\}$,

(v) $\left\{\mathscr{C}\left(2^{\omega}, 0,1\right)\right\}$,

(vi) $\left\{\mathscr{C}\left(2^{\omega}, 1,1\right)\right\}$.

In Chapter 1 we consider orbitally stable attractors of uniform flows in general. In $\S 1.1$ we explain the main results of this paper, some of which follow.

THEOREM 1. If $A \subset T$ is an orbitally stable attractor of a uniform $C^{0}$ flow, then the class of $A$ is permissible.

THEOREM 2. For any fixed permissible class there exists a uniform $C^{\infty}$ flow possessing an orbitally stable attractor of that class.

Theorem 3 details necessary dynamical features for a given set $A \subset T$ to be an orbitally stable attractor of a given uniform $C^{0}$ flow $\varphi_{t}$. In particular, if $A$ is an orbitally stable attractor of $\varphi_{t}$, then there exists a sequence $\left\{T^{n}\right\}_{n=0}^{\infty}$ of strictly decreasing (Definition 8) positively invariant uniform open solid tori (Definition 7) such that $A=\operatorname{Fr}\left(\cap T^{n}\right)$.

Theorem 4 states that those necessary conditions of Theorem 3 which apply nontrivially are also sufficient.

In $§ 1.2$ we prove some useful lemmas using point set topology.

In $\$ 1.3$ we prove Theorems 1,3 , and 4 .

In $\$ 1.4$ we investigate the first return map $\varphi_{2 \pi}$ of a given uniform $C^{0}$ flow $\varphi_{t}$ on the cross-section $A_{\theta}$ of a given orbitally stable attractor $A$ on the space of components of $A_{\theta}$. In particular, we find that if the class of $A$ is $\mathscr{C}\left(2^{\omega}, 0,1\right)$, then the map $\varphi_{2 \pi}: A_{\theta} \rightarrow A_{\theta}$ is conjugate to an irrational rotation on a certain abelian Cantor (compact, perfect, and totally disconnected) group $\Lambda$. That is, there exists a homeomorphism $\psi: \Lambda \rightarrow A_{\theta}$ and an irrational rotation $f: \Lambda \rightarrow \Lambda$ such that $\varphi_{2 \pi}$ : $\psi \circ f \circ \psi^{-1}$.

We also consider the following type of set. 
DEFINITION 6. A set $K \subset T$ is called a circular solenoid if $K=\bigcap_{n=1}^{\infty} T^{n}$, where each $T^{n}=\left(\tau_{0} \circ \tau_{1} \circ \cdots \circ \tau_{n-1}\right)(T)$ and each circular solenoidal map $\tau_{k}: \bar{T} \rightarrow T$ is defined by

$$
\tau_{k}(z, \theta)=\left(r_{k} z+s_{k} \exp \left(i l_{k} \theta\right), m_{k} \theta\right),
$$

where $r_{k}, s_{k} \in \mathbf{C}, l_{k}, m_{k} \in \mathbf{Z}, l_{k} \neq 0, m_{k} \geqslant 2$ are chosen so that $\tau_{k}$ is injective, and $\operatorname{gcd}\left(l_{k}, m_{k}\right)=1$ (otherwise $\tau_{k}(T)$ is not connected). The standard solenoid $L$ is the circular solenoid where each of the $\tau_{k}$ is the standard solenoidal map $\tau: \bar{T} \rightarrow T$ defined by

$$
\tau(z, \theta)=(z / 4+(1 / 2) \exp (i \theta), 2 \theta) .
$$

The standard solenoid is a well-known example of an attractor of a three-dimensional map; cf. [Bowen, 1978] (Figure 1). Also, the cross-section is an example of an attractor of a $C^{1}$ diffeomorphism [Bowen and Franks, 1976].

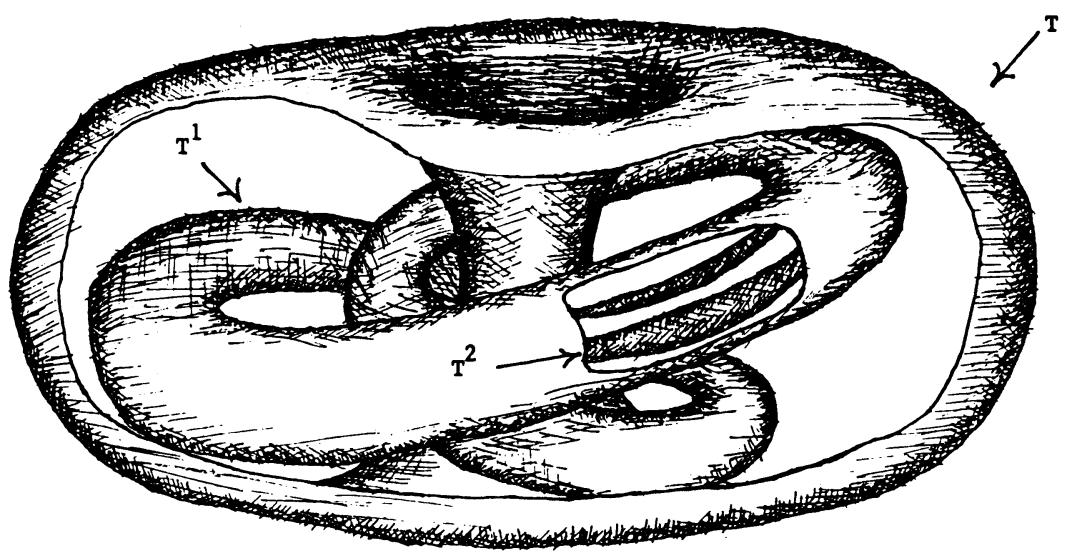

(a) Cut-away view

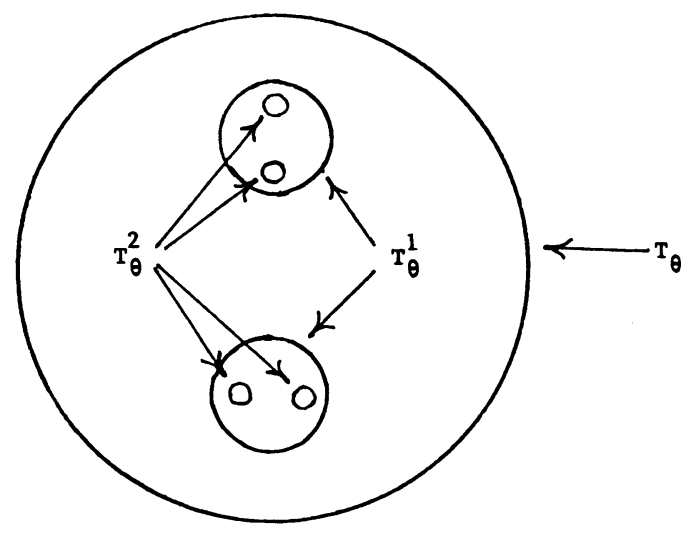

(b) Cross-section

Figure 1. The nested open solid tori $T, T^{1}$ and $T^{2}$ 
Each $T^{n}$ is an open solid torus with winding number $\prod_{k=0}^{n-1} m_{k}$, and each $T_{\theta}^{n}$ consists of $\prod_{k=0}^{n-1} m_{k}$ disjoint disks of radius $\prod_{k=0}^{n-1}\left|r_{k}\right|$. Also, the set $K_{\theta}$ is a Cantor set.

We explicitly calculate the first return map $\varphi_{2 \pi}: K_{\theta} \rightarrow K_{\theta}$ for any uniform $C^{0}$ flow $\varphi_{t}$ for which the circular solenoid $K$ is invariant. Using Taylor's theorem we prove Theorem 6, which states that the standard solenoid $L$ is not an invariant set of any uniform $C^{2}$ flow. This indicates that the construction of class $\mathscr{C}\left(2^{\omega}, 0,1\right)$ orbitally stable attractors of uniform $C^{\infty}$ flows requires a special construction.

In Chapter 2 we prove Theorem 2 by constructing the required examples of uniform $C^{\infty}$ flows possessing orbitally stable attractors. We comment on some measure-theoretic properties of these attractors.

In $\S 2.1$ we consider the collections of classes $\mathscr{C}(N, 0,1), \mathscr{C}(N, 1,1), \mathscr{C}(N, 1, M)$ and $\mathscr{C}(N, 1, \omega)$. For any $N \in \mathbf{Z}^{+}$, construction of orbitally stable attractors in classes $\mathscr{C}(N, 0,1)$ and $\mathscr{C}(N, 1,1)$ is not difficult. We briefly present the Plykin attractor [Plykin, 1974; Newhouse, 1980; Guckenheimer and Holmes, 1983] and some variations, as examples of orbitally stable attractors of classes $\mathscr{C}(N, 1, M), N, M \in$ $\mathbf{Z}^{+}, M \geqslant 2$, which display interesting dynamics. We then show how the "Lakes of Wada" construction [Yoneyama, 1917; Hocking and Young, 1961] may be used to construct orbitally stable attractors of uniform $C^{\infty}$ flows of classes $\mathscr{C}(N, 1, \omega)$, $N \in \mathbf{Z}^{+}$.

In $\$ 2.2$ we consider the problem of imbedding circular solenoids as orbitally stable attractors of uniform $C^{k}$ flows. Any such attractor is of class $\mathscr{C}\left(2^{\omega}, 0,1\right)$. Theorem 7 describes sufficient conditions on circular solenoids so that they can be imbedded as orbitally stable attractors of uniform $C^{k}$ flows. As a corollary to Theorem 7 we find that there exist circular solenoids which can be imbedded as orbitally stable attractors of uniform $C^{\infty}$ flows.

In $\$ 2.3$ we use noncircular solenoid-like maps to construct examples of uniform $C^{\infty}$ flows possessing orbitally stable attractors of class $\mathscr{C}\left(2^{\omega}, 1,1\right)$. One of these attractors has positive Lebesgue 3-measure.

In $\$ 2.4$ we consider the Hausdorff dimension; cf. [Kahane and Salem, 1963] of orbitally stable attractors of uniform Lipschitz flows. We show that for any $\delta \in[1,3]$, there exists a uniform $C^{\infty}$ flow possessing an orbitally stable attractor whose Hausdorff dimension is $\delta$.

ACKNowledgement. I would like to thank Julian Palmore and Felix Albrecht for their guidance.

\section{Chapter 1. Orbitally Stable attractors}

1.1 Main results. Consider an orbitally stable attractor $A$ of a uniform $C^{0}$ flow $\varphi_{t}$. For any $\varepsilon>0$ there exists an open neighborhood $V_{\varepsilon}$ of $A$ such that $\varphi_{t}\left(V_{\varepsilon}\right) \subset N(A, \varepsilon)$ for all $t>0$. So the set

$$
S_{\varepsilon}=\bigcup_{0<t} \varphi_{t}\left(V_{\varepsilon}\right)
$$


is a positively invariant open neighborhood of $A$, contained in $N(A, \varepsilon)$. The fact that the flow is uniform and the manifold is $T$ allows us, in the proofs of Theorems 1 and 4 , to fill in holes and otherwise modify $S_{\varepsilon}$ to construct a positively invariant set $T_{\varepsilon} \supset A$, such that $\operatorname{Fr}\left(T_{\varepsilon}\right) \subset \overline{N(A, \varepsilon)}$, and $T_{\varepsilon}$ is a uniform open solid torus.

Definition 7. A set $S \subset T$ is called a uniform open solid torus if $S$ is the homeomorphic image of the standard open solid torus $T$ such that for each $\theta \in[0,2 \pi)$ the number of components of $T_{\theta} \cap S$ is $N \in \mathbf{Z}^{+}$and each component is homeomorphic to the open planar disk. We denote the winding number of $S$ by $\#(S)=N$.

Notice that although a uniform open solid torus $S$ is homeomorphic to $T$, it is not in general true that $\bar{S}$ is homeomorphic to $\bar{T}$. Also, if $T^{n+1} \subset T^{n}$, where $T^{n}, T^{n+1}$ are uniform open solid tori, then $\#\left(T^{n+1}\right)=k \#\left(T^{n}\right)$ for some $k \in \mathbf{Z}^{+}$.

DEFINITION 8. A sequence of subsets of $T,\left\{E^{n}\right\}_{n=0}^{\infty}$, is called strictly decreasing if, for each $n, \overline{E^{n+1}} \subset \operatorname{INT}\left(E^{n}\right)$. It is called strictly increasing if, for each $n$, $\operatorname{INT}\left(E^{n+1}\right) \supset \overline{E^{n}}$.

Definition 9. A $T$-sequence for a set $A \subset T$ is a strictly decreasing sequence of uniform open solid tori $\left\{T^{n}\right\}_{n=0}^{\infty}$ such that $A=\operatorname{Fr}\left(\cap T^{n}\right)$. The $T$-sequence is called positively invariant if each $T^{n}$ is positively invariant under the flow. The $T$-sequence is called solenoidal if $\lim \#\left(T^{n}\right)=\infty$. Any set which has a solenoidal $T$-sequence is called a solenoid or a solenoidal set.

Definition 10. An I-sequence for a uniform open solid torus $S \subset T$ is a strictly increasing sequence of uniform open solid tori $\left\{I^{k}\right\}_{k=0}^{\infty}$ such that $S=U I^{k}$. The $I$-sequence is called negatively invariant if each $I^{k}$ is negatively invariant under the flow.

In Theorems 1, 3 and 4, we consider various conditions which can be placed on a given positively invariant $T$-sequence $\left\{T^{n}\right\}_{n=0}^{\infty}$ for a given set $A$. We find that if $\left\{T^{n}\right\}$ satisfies certain combinations of these conditions, and the flow is uniform Lipschitz, then $A$ is an orbitally stable attractor. We also find that if $A$ is an orbitally stable attractor and the flow is uniform $C^{0}$, then we can find such a $T$-sequence for $A$.

DEFINITION 11. The following are conditions on a given positively invariant $T$-sequence $\left\{T^{n}\right\}_{n=0}^{\infty}$ :

(a) For each $n \geqslant 0$, almost every point in $T^{n} \backslash T^{n+1}$ flows eventually into $T^{n+1}$. (By the positive invariance of $T^{n+1}$, one never flows out.)

(b) For any $\varepsilon>0$ and $n \geqslant 0$, there exists $m \geqslant n$ such that almost every point in $T^{n} \backslash T^{n+1}$ flows to within $\varepsilon$ of every point of $\operatorname{Fr}\left(T^{m}\right)$.

(c) The set $G=\operatorname{INT}\left(\cap T^{n}\right)$ is the disjoint union of the uniform open solid tori $\left\{I_{k}\right\}$ where $k \in \mathbf{Z}^{+}, k \leqslant @(G)$, and for each $k$ we have $\operatorname{Fr}\left(I_{k}\right)=A$, and $I_{k}$ has a negatively invariant $I$-sequence $\left\{I_{k}^{n}\right\}_{n=0}^{\infty}$ such that for any $\varepsilon>0$ and $n \geqslant 0$, there exists $m \geqslant n$ such that almost every point in $I_{k}^{n+1} \backslash I_{k}^{n}$ flows eventually out of $I_{k}^{n+1}$ and to within $\varepsilon$ of every point of $\operatorname{Fr}\left(I_{k}^{m}\right)$.

(d) For all but finitely many $k \in \mathbf{Z}^{+}, k \leqslant @(G)$, almost every point in $I_{k}^{0}$ (as in (c)) flows eventually out of $I_{k}^{0}$.

(e) The diameters of the components of the $T^{n}$ go uniformly to 0 as $n$ goes to $\infty$.

$(\neg$ e) The negation of condition (e). 
THEOREM 1. If $A \subset T$ is an orbitally stable attractor of a uniform $C^{0}$ flow, then the class of $A$ is permissible.

THEOREM 2. For any fixed permissible class there exists a uniform $C^{\infty}$ flow possessing an orbitally stable attractor of that class.

THEOREM 3. If the set $A \subset T$ is an orbitally stable attractor of a uniform $C^{0}$ flow $\varphi_{t}$, then there exists a positively invariant T-sequence $\left\{T^{n}\right\}_{n=0}^{\infty}$ for $A$ so that we have:

(1)

$$
\lim _{n \rightarrow \infty} \#\left(T^{n}\right)= \begin{cases}\infty & \text { if } @\left(A_{\theta}\right)=2^{\omega}, \\ @\left(A_{\theta}\right) & \text { otherwise. }\end{cases}
$$

(2) Condition (e) or $(\neg \mathrm{e})$ holds, depending on whether the second term of class $(A)$ is 0 or 1 , respectively.

(3) @(INT $\left.\left(\cap T^{n}\right)\right)=@(C A)-1($ here $\omega-1=\omega)$.

(4) Conditions (a), (b), (c) and (d) hold.

Note 1 . If class $(A) \notin\left\{\mathscr{C}(N, 1, \omega) \mid N \in \mathbf{Z}^{+}\right\}$, then condition (d) holds trivially. If

$$
\text { class }(A) \notin\left\{\mathscr{C}(N, 1, M) \mid N, M \in \mathbf{Z}^{+}, M \geqslant 2\right\} \cup\left\{\mathscr{C}(N, 1, \omega) \mid N \in \mathbf{Z}^{+}\right\} \text {, }
$$

then condition (c) holds trivially.

THEOREM 4. Let $\varphi_{t}$ be a uniform $C^{0}$ flow whose induced measures are absolutely continuous with respect to normalized Lebesgue measure $\mathscr{M}\left(i . e . \mathscr{M}\left(\varphi_{t}(E)\right)=0\right.$ if and only if $\mathscr{M}(E)=0 \forall t \in \mathbf{R} \forall E \subset T)$. Then the set $A \subset T$ is an orbitally stable attractor of $\varphi_{t}$ and class $(A)$ respectively belongs to collection (i), (ii), (iii), (iv), (v), or (vi) of Definition 5 if and only if there exists a positively invariant $T$-sequence $\left\{T^{n}\right\}_{n=0}^{\infty}$ for $A$ so that we have (i'), (ii'), (iii'), (iv'), ( $\left.{ }^{\prime}\right)$, or (vi'), respectively, where:

(i') $\lim _{n \rightarrow \infty} \#\left(T^{n}\right) \in \mathbf{Z}^{+}$, and conditions (a) and (e) hold.

(ii') $\lim _{n \rightarrow \infty} \#\left(T^{n}\right) \in \mathbf{Z}^{+}, \operatorname{INT}\left(\cap T^{n}\right)=\varnothing$, and conditions (a), (b) and ( $\neg$ e) hold.

(iii') $\lim _{n \rightarrow \infty} \#\left(T^{n}\right) \in \mathbf{Z}^{+}, @\left(\operatorname{INT}\left(\cap T^{n}\right)\right) \in \mathbf{Z}^{+}$, and conditions (a), (b), (c) and ( $\neg$ e) hold.

(iv') $\lim _{n \rightarrow \infty} \#\left(T^{n}\right) \in \mathbf{Z}^{+}$, @(INT $\left.\left(\cap T^{n}\right)\right)=\omega$, and conditions (a), (b), (c), (d) and $(\neg \mathrm{e})$ hold.

$\left(\mathrm{v}^{\prime}\right) \lim _{n \rightarrow \infty} \#\left(T^{n}\right)=\infty$, and conditions (a) and (e) hold.

(vi') $\lim _{n \rightarrow \infty} \#\left(T^{n}\right)=\infty$, and conditions (a), (b) and $(\neg \mathrm{e})$ hold.

Note 2. If $\varphi_{t}$ is Lipschitz, then the induced measures are absolutely continuous.

1.2 Properties of $T$-sequences. We prove some lemmas about $T$-sequences using point set topology. These lemmas are used in $\S 1.3$ to help prove Theorems 1,3 and 4 , and are also used in $\$ 1.4$ when we investigate first return maps.

Lemma 1. Let $\left\{T^{n}\right\}_{n=0}^{\infty}$ be a T-sequence for a set $A \subset T$. Then the set $E_{\theta}$ is a component of $A_{\theta}$ if and only if there exists a strictly decreasing sequence of sets $\left\{E_{\theta}^{n}\right\}$ such that $E_{\theta}=\operatorname{Fr}\left(\cap_{n=0}^{\infty} E_{\theta}^{n}\right)$ and each $E_{\theta}^{n}$ is a component of $T_{\theta}^{n}$. 
Proof of Lemma 1. The set $F_{\theta}$ is a component of $\cap T_{\theta}^{n}$ if and only if there exists a strictly decreasing sequence of sets $\left\{F_{\theta}{ }^{n}\right\}$ such that $F_{\theta}=\bigcap F_{\theta}^{n}$ and each $F_{\theta}^{n}$ is a component of $T_{\theta}^{n}$. Any component of $\cap T_{\theta}^{n}$ is simply connected, and so must have a connected frontier. The set $\cap T_{\theta}^{n}$ is closed since $\left\{T^{n}\right\}$ is strictly decreasing, so $\operatorname{Fr}\left(\cap T^{n}\right)=A_{\theta}$ is the union of the frontiers of its components.

Note 3. Suppose that $\left\{T^{n}\right\}$ is a $T$-sequence for $A$. Then Lemma 1 implies that

$$
\lim _{n \rightarrow \infty} \#\left(T^{n}\right)= \begin{cases}\infty & \text { if } @\left(A_{\theta}\right)=2^{\omega}, \\ @\left(A_{\theta}\right) & \text { otherwise. }\end{cases}
$$

LEMMA 2. Let $\left\{E^{n}\right\}_{n=0}^{\infty}$ be a strictly decreasing sequence of open sets in either $T$ or $T_{\theta}$. Then for any $\varepsilon>0$, there exists an $M \in \mathbf{Z}^{+}$such that $m \geqslant M$ implies $E^{m} \subset$ $N\left(\cap E^{n}, \varepsilon\right)$.

Proof of Lemma 2. Suppose not, then choose a sequence $\left\{X^{m}\right\}_{m=0}^{\infty}$ such that for each $m \geqslant 0, x^{m} \in E^{m}$ and $d\left(x^{m}, \cap E^{n}\right) \geqslant \varepsilon>0$. Thus $\left\{X^{m}\right\}$ has an accumulation point outside $N\left(\cap E^{n}, \varepsilon\right)$. Since the sequence $\left\{E^{n}\right\}$ is strictly decreasing, we have $\cap E^{n}=\cap \overline{E^{n}}$ and all accumulation points of $\left\{X^{m}\right\}$ must be contained in $\cap E^{n}$. This contradiction completes the proof.

LEMMA 3. Let $\left\{E^{n}\right\}_{n=0}^{\infty}$ be a strictly decreasing sequence of open sets in either $T$ or $T_{\theta}$. Then for any $\varepsilon>0$, there exists an $M \in \mathbf{Z}^{+}$such that $m \geqslant M$ implies $\operatorname{Fr}\left(\cap E^{n}\right) \subset$ $N\left(\operatorname{Fr}\left(E^{m}\right), \varepsilon\right)$.

Proof of Lemma 3. For any $x \in \operatorname{Fr}\left(\cap E^{n}\right)$, there exists a sequence of points $\left\{x^{m}\right\}_{m=0}^{\infty}$ such that each $x^{m} \in \operatorname{Fr}\left(E^{m}\right)$ and $\lim x^{m}=x$. Thus for any $\varepsilon>0$ there exists an $M_{\varepsilon}(x) \in \mathbf{Z}^{+}$such that $m>M_{\varepsilon}(x)$ implies $d\left(x^{m}, x\right)<\varepsilon$. By the compactness of $\operatorname{Fr}\left(\cap E^{n}\right)$, Lemma 3 is true.

Lemma 4. Let $\left\{T^{n}\right\}_{n=0}^{\infty}$ be a T-sequence for $A$. Then the components of $A_{\theta}$ are all singleton points if and only if the diameters of the components of $T_{\theta}^{n}$ go uniformly to 0 as $n$ goes to $\infty$.

Proof of Lemma 4. The "if" implication follows immediately from Lemma 1.

If the diameters of the components of $T_{\theta}^{n}$ do not go uniformly to 0 , then there exists a strictly decreasing sequence of sets $\left\{E_{\theta}^{n}\right\}$ such that each $E_{\theta}^{n}$ is a component of $T_{\theta}^{n}$ and $\lim \operatorname{diam}\left(E_{\theta}^{n}\right)>0$. But

$$
\operatorname{diam}\left(\operatorname{Fr}\left(\cap E_{\theta}^{n}\right)\right)=\operatorname{diam}\left(\cap E_{\theta}^{n}\right)=\operatorname{diam}\left(\cap \overline{E_{\theta}^{n}}\right)>0,
$$

so by Lemma 1, the "only if" implication holds.

LemMa 5. Let $\varphi_{t}$ be a uniform $C^{0}$ flow, and let $E \subset T$ be a positively invariant open neighborhood of a nonempty compact connected invariant set $A$ such that every component of $E_{\theta}$ has nonempty intersection with $A_{\theta}$. Then $\varphi_{2 \pi}$ maps the components of $E_{\theta}$ into each other in a cyclic manner. 
Proof of Lemma 5. The set $E_{\theta}$ has finitely many components. Suppose $\varphi_{2 \pi}$ does not map the components of $E_{\theta}$ into each other in a cyclic manner. Then the invariance of $A_{\theta}$ implies a product of cycles. Thus $\bigcup_{0<t \leqslant 2 \pi} \varphi_{t}\left(E_{\theta}\right)$, which is an open neighborhood of $A$, has at least two components. This contradicts the connectedness of $A$.

LEMMA 6. Let $\varphi_{t}$ be a uniform $C^{0}$ flow. If $A$ is an orbitally stable attractor, and $\left\{T^{n}\right\}_{n=0}^{\infty}$ is a $T$-sequence for $A$, then the components of the set $G_{\theta}=\operatorname{INT}\left(\cap_{n=0}^{\infty} T_{\theta}^{n}\right)$ are each homeomorphic to the open planar disk and the map $\varphi_{2 \pi}$ permutes these components as a disjoint product of finite cycles.

Proof of Lemma 6. The map $\varphi_{2 \pi}$ permutes the components of $G_{\theta}$, so it acts as a disjoint product of cycles. Suppose one of these cycles is infinite, and $\mathcal{O}_{\theta}^{0}$ is a particular component of $G_{\theta}$ in this cycle. Then the elements of the cycle are $\left\{\mathcal{O}_{\theta}^{n} \mid n \in \mathbf{Z}\right\}$, where $\mathcal{O}_{\theta}^{n}=\varphi_{2 \pi n}\left(\mathcal{O}_{\theta}^{0}\right)$. Choose a point $p \in \mathcal{O}_{\theta}^{0}$, and let $\varepsilon$ denote the distance from $p$ to $\operatorname{Fr}\left(\mathcal{O}_{\theta}^{0}\right)\left(\varepsilon=d\left(p, \operatorname{Fr}\left(\mathcal{O}_{\theta}^{0}\right)\right)=d\left(p, A_{\theta}\right)\right)$.

By the orbital stability of $A$ there exists a $\delta>0$ such that for any point $q \in N(A, \delta)$ we have

$$
d\left(\varphi_{2 \pi n}(q), A_{\theta}\right)<\varepsilon \text { for all } n \in \mathbf{Z}^{+} .
$$

The sets $\left\{\mathcal{O}_{\theta}^{n}\right\}_{n=0}^{\infty}$ are all mutually disjoint, and the sets $\left\{\operatorname{Fr}\left(\mathcal{O}_{\theta}^{n}\right)\right\}_{n=0}^{\infty}$ are all contained in $A_{\theta}$. Thus there exists an $m \leqslant 0$ such that $\mathcal{O}_{\theta}^{m} \subset N(A, \delta)$. But the point $\varphi_{2 \pi m}(p)$ is contained in $\mathcal{O}_{\theta}^{m}$ and thus also in $N(A, \delta)$, while $\varphi_{-2 \pi m}\left(\varphi_{2 \pi m}(p)\right)=p$ is a distance $\varepsilon$ away from $A_{\theta}$. This contradicts the orbital stability of $A$.

Each component of $G_{\theta}$ is a component of $C A_{\theta}$, and $A_{\theta}$ is compact. So, each component of $G_{\theta}$ is homeomorphic to the open planar disk.

Note 4. Lemmas 1, 5 and 6 imply that if $\left\{T^{n}\right\}$ is a $T$-sequence for an orbitally stable solenoidal attractor $A$ of a uniform $C^{0}$ flow, then $\cap T^{n}$ has an empty interior and $C A$ has exactly one component.

\subsection{Proof of Theorems 1,3 and 4.}

Proof of Theorems 1 AND 3. Let $A \subset T$ be an orbitally stable attractor of $\varphi_{t}$. We now construct a sequence of positively invariant uniform open solid tori $\left\{S^{n}\right\}_{n=0}^{\infty}$, so that $S^{n} \supset A, S^{n} \subset \mathscr{B}(A)$, and $\operatorname{Fr}\left(S^{n}\right) \subset\left(N\left(A, 2^{-n}\right) \cup T_{\theta}\right)$.

Let $U^{n}$ denote an open neighborhood of $A$, so that $U^{n} \subset \mathscr{B}(A)$, and $U^{n} \subset$ $N\left(A, 2^{-n}\right)$. By the orbital stability of $A$, there exists an open neighborhood $V^{n}$ of $A$, so that $\varphi_{t}\left(V^{n}\right) \subset U^{n} \forall t>0$. Let $W^{n}$ denote $\bigcup_{0<t} \varphi_{t}\left(V^{n}\right)$. The set $W^{n}$ is a positively invariant open neighborhood of $A$, and $W^{n} \subset U^{n}$.

Let $X_{\theta}^{n}$ denote the union of all components of $W_{\theta}^{n}$ which have nonempty intersection with $A$. The components of $X_{\theta}^{n}$ need not be simply connected, so we consider $Y_{\theta}^{n}$, which is $X_{\theta}^{n}$ with all the holes filled in. More precisely,

$$
Y_{\theta}^{n}=C \quad \text { (unbounded component of } C X_{\theta}^{n} \text { ). }
$$

Each component of $Y_{\theta}^{n}$ is open and simply connected, and thus is homeomorphic to the open planar disk. Let $S^{n}$ denote

$$
\bigcup_{0<t \leqslant 2 \pi} \varphi_{t}\left(Y_{\theta}^{n}\right)
$$


By Lemma 5 we see that $S^{n}$ is connected, and one can show [Edwards, 1962] that $S^{n}$ is a uniform open solid torus. Notice that $\operatorname{Fr}\left(S^{n}\right)$ is not necessarily homeomorphic to $\operatorname{Fr}(T)$. Also, we have

$$
\operatorname{Fr}\left(S^{n}\right)=\left(\bigcup_{0<t \leqslant 2 \pi} \varphi_{t}\left(\operatorname{Fr}\left(Y_{\theta}^{n}\right)\right)\right) \cup\left(Y_{\theta}^{n} \backslash \varphi_{2 \pi}\left(Y_{\theta}^{n}\right)\right),
$$

and, since $\operatorname{Fr}\left(Y_{\theta}^{n}\right) \subset \operatorname{Fr}\left(X_{\theta}^{n}\right)$, we have

$$
\operatorname{Fr}\left(S^{n}\right) \subset\left(N\left(A, 2^{-n}\right) \cup T_{\theta}\right) .
$$

Suppose $A \not \supset \operatorname{Fr}\left(\cap S^{n}\right)$, then there exist $x \in C A$ such that $x \in \operatorname{Fr}\left(\cap S^{n}\right)$. Let $\left\{x_{n}\right\}$ be a sequence of points such that $x_{n} \in C S^{n}$ and $d\left(x_{n}, x\right)=d\left(C S^{n}, x\right)$. Each $x_{n} \in \operatorname{Fr}\left(S^{n}\right)$ and $\lim x_{n}=x$. If

$$
x_{n} \in \bigcup_{0 \leqslant t \leqslant 2 \pi} \varphi_{t}\left(\operatorname{Fr}\left(Y_{\theta}^{n}\right)\right)
$$

for infinitely many $n$, then since $\varphi_{t}$ is uniformly continuous over $t \in[0,2 \pi]$ and $\operatorname{Fr}\left(Y_{\theta}^{n}\right) \subset N\left(A_{\theta}, 2^{-n}\right)$ we have that $x$ must be contained in $A$. But $x \in C A$, so $x_{n} \in Y_{\theta}^{n} \backslash \varphi_{2 \pi}\left(Y_{\theta}^{n}\right)$ for infinitely many $n$, thus $x \in T_{\theta}$, and those $x_{n}$ in $T_{\theta}$ must also be contained in their respective $\varphi_{2 \pi}\left(\operatorname{Fr}\left(Y_{\theta}^{n}\right)\right)$. This is a contradiction to $A \not \supset \operatorname{Fr}\left(\cap S^{n}\right)$, so $A \supset \operatorname{Fr}\left(\cap S^{n}\right)$.

Now select $\left\{T^{m}\right\}$ to be a subsequence of $\left\{S^{n}\right\}$ which is strictly decreasing. Since $\cap S^{n}=\cap T^{m}$, we have $A \supset \operatorname{Fr}\left(\cap T^{m}\right)$.

Suppose $\operatorname{Fr}\left(\cap T^{m}\right) \not \supset A$, then, since $A \subset\left(\cap T^{m}\right)$ there exists an $x \in A$ such that $x \in \operatorname{INT}\left(\cap T^{m}\right)=G_{\theta}$. Let the point $p \in T^{0} \backslash T^{1}$ be such that $\omega(p) \supset x$. Then let $\left\{t_{m}\right\}$ be a sequence of times such that $\varphi_{t_{m}}(p) \in T^{m} \backslash T^{m+1}$. Since the sequence $\left\{T^{m}\right\}$ is a strictly decreasing sequence of positively invariant sets, the $\left\{t_{m}\right\}$ exist and are increasing. If $\lim t_{m}=t_{\infty}<\infty$, then,

$$
\varphi_{t_{\infty}}(p) \in \operatorname{Fr}\left(\cap T^{m}\right) \subset A .
$$

This contradicts the invariance of $A$. If $\lim t_{m}=\infty$, then $\omega(p) \not \supset x$, since $x \in G$. But $x \in A=\omega(p)$, a contradiction to $\operatorname{Fr}\left(\cap T^{m}\right) \not \supset A$, so $\operatorname{Fr}\left(\cap T^{m}\right) \supset A$.

Fix $\varepsilon / 2>0$ and $m \geqslant 0$. Then by Lemma 2 for some $k \geqslant m+1$, we have $B(A, \varepsilon / 2) \supset \operatorname{Fr}\left(T^{k}\right)$. For almost every point $p \in T^{m} \backslash T^{m+1}$ we have $\omega(p)=A$, so any such $p$ flows into $T^{m+1}$ and to within $\varepsilon$ of every point in $T^{k}$. Thus conditions (a) and (b) are necessary for any orbitally stable attractor of a uniform $C^{0}$ flow. By Lemma 4, condition (e) is necessary if class $(A) \in \mathscr{C}(\cdot, 0, \cdot)$, and condition $(\neg \mathrm{e})$ is necessary if class $A \in \mathscr{C}(\cdot, 0, \cdot)$. By Lemma $1, \lim \#\left(T^{n}\right)=N \in \mathbf{Z}^{+}$if class $(A) \in \mathscr{C}(N, \cdot, \cdot)$, and $\lim \#\left(T^{n}\right)=\infty$ if class $(A) \in \mathscr{C}\left(2^{\omega}, \cdot, \cdot\right)$. By Note 4 , we have @ $(C A)=1$ if class $(A) \in \mathscr{C}\left(2^{\omega}, \cdot, \cdot \cdot\right)$.

Consider any component $H_{\theta}$ of $G_{\theta}$. By Lemma 6, the set $H_{\theta}$ is homeomorphic to the open planar disk, and there exists a $k \in \mathbf{Z}^{+}$such that $\varphi_{2 \pi k}\left(H_{\theta}\right)=H_{\theta}$. The set

$$
I=\bigcup_{0 \leqslant t<2 \pi k} \varphi_{t}\left(H_{\theta}\right)
$$

is a uniform open solid torus and \#(I) divides $k$. So each component of $G$ is a uniform open solid torus. 
For some $M \in \mathbf{Z}^{+}$sufficiently large, the set $I \backslash N\left(A, 2^{-M}\right)$ contains a simple closed curve $J$ with winding number $\#(I)$. For each $n>M$, let $Y_{\theta}^{n}$ be the union of the \#(I) components of $C \overline{X_{\theta}^{n}}$ which have nonempty intersection with $J$. The rest of the construction of the negatively invariant $I$-sequence such that (c) holds is similar to the construction of the $T$-sequence such that (a) and (b) hold.

Condition (d) is necessary since otherwise $\mathscr{B}(A)$ does not contain a neighborhood of $A$.

Proof of Theorem 4. The "only if" implication follows from Theorem 3.

Let $\varphi_{t}$ be a uniform $C^{0}$ flow whose induced measures are absolutely continuous with respect to Lebesgue 3-measure. Assume that a positively invariant $T$-sequence $\left\{T^{n}\right\}_{n=0}^{\infty}$ for $A \subset T$ satisfies condition (a). For each choice of $m, k \in \mathbf{Z}, m \geqslant k \geqslant 0$, let $E_{m}^{k}$ be the set of all points in $T^{k} \backslash T^{k+1}$ which eventually flow into $T^{m}$, but which never flow into $T^{m+1}$.

By condition (a), and the absolute continuity of the measures induced by $\varphi_{t}$, we have $\mathscr{M}\left(E_{m}^{k}\right)=0$ for all $m, k \in \mathbf{Z}^{+} \cup\{0\}, m \geqslant k$. So almost every point in $T^{k}$ flows eventually into $T^{m}$ for all $m \in \mathbf{Z}^{+} \cup\{0\}$.

If condition (e) also holds, then condition (b) holds.

If condition (b) holds, we now have that for any $\varepsilon>0$ and $M<\infty$, almost every point in $T^{0} \backslash \cap T^{n}$ flows to within $\varepsilon$ of every point of $\operatorname{Fr}\left(T^{m}\right)$ for some $m>M$. By Lemma 3, almost every point in $T^{0} \backslash \cap T^{n}$ has $A$ as its $\omega$-limit set. Similarly, if condition (c) holds, then almost every point in the set

$$
\cup I_{k} \backslash I_{k}^{0}, \quad k \in \mathbf{Z}^{+} \cup\{0\}, k<@(G),
$$

has $A$ as its $\omega$-limit set.

If condition (d) holds, then $\mathscr{B}(A)$ contains a deleted neighborhood of $A$.

Lemma 4 shows that conditions (e) or $(\neg \mathrm{e})$ hold if and only if class $(A)=\mathscr{C}(\cdot, P, \cdot)$ or $\mathscr{C}(\cdot, 0, \cdot)$, respectively.

1.4 Dynamics of the first return map. Let $A$ be an orbitally stable attractor of the uniform $C^{0}$ flow $\varphi_{t}$. The map $\varphi_{2 \pi}$ permutes the components of $A_{\theta}$. Let $\sim$ be the equivalence relation on $A_{\theta}$ which identifies the points in each component of $A_{\theta}$. Let the map $\bar{\varphi}: A_{\theta} / \sim \rightarrow A_{\theta} / \sim$ be defined by

$$
\bar{\varphi}=\pi \circ \varphi_{2 \pi} \circ \pi^{-1},
$$

where $\pi: A_{\theta} \rightarrow A_{\theta} / \sim$ is the canonical projection. If $A$ is solenoidal, then we find that $\bar{\varphi}$ is conjugate to an irrational rotation on a certain compact abelian group.

Also, we explicitly calculate the map $\varphi_{2 \pi}$ when $A$ is a circular solenoid, and show that the usual solenoid $L$ cannot be imbedded as an orbitally stable attractor of a uniform $C^{2}$ flow.

THEOREM 5. If $A$ is an invariant solenoid of a uniform $C^{0}$ flow $\varphi_{t}$, then the map $\bar{\varphi}$ : $A_{\theta} / \sim \rightarrow A_{\theta} / \sim$ is conjugate to an irrational rotation on a compact perfect totally disconnected abelian group.

We state the following lemma without proof. 
LEMMA 7. Let $\varphi_{t}$ be a uniform $C^{0}$ flow and let $E \subset T$ be a uniform open solid torus containing a compact invariant set $A$. Let $\approx$ be the equivalence relation on $A_{\theta}$ which identifies the points in each component of $E_{\theta}$. Let the map $\overline{\bar{\varphi}}: A_{\theta} / \approx \rightarrow A_{\theta} / \approx$ be defined by

$$
\overline{\bar{\varphi}}=\bar{\pi} \circ \varphi_{2 \pi} \circ \bar{\pi}^{-1},
$$

where $\bar{\pi}: A_{\theta} \rightarrow A_{\theta} / \approx$ is the canonical projection. Then $A_{\theta} / \approx$ has $\#(E)$ elements, and $\overline{\bar{\varphi}}$ cyclically permutes them.

Proof of Theorem 5. Let $\left\{T^{n}\right\}$ be a $T$-sequence for $A$. Let $\Lambda$ denote the space of all infinite sequences, $\lambda=\lambda_{0}, \lambda_{1}, \ldots$, where $\lambda_{k} \in \mathbf{Z} \bmod \left(\#\left(T^{k}\right) / \#\left(T^{k-1}\right)\right)$ for $k \in \mathbf{Z}^{+}$and $\lambda_{0} \in \mathbf{Z} \bmod \left(\#\left(T^{0}\right)\right)$. The group operation $\oplus$ on $\Lambda$ is defined by

$$
(\alpha \oplus \beta)_{n}=I \sum_{k=1}^{n}\left(\alpha_{k}+\beta_{k}\right)\left(\#\left(T^{k}\right)\right) /\left(\#\left(T^{n}\right)\right) I \bmod \left(\left(\#\left(T^{n}\right)\right) /\left(\#\left(T^{n-1}\right)\right)\right),
$$

where $I \cdot I$ denotes the greatest integer. The translation invariant metric $|\cdot, \cdot|$ is defined by

$$
|\alpha, \beta|=\sum_{k=1}^{\infty}\left(\left(\alpha \oplus \beta^{-1}\right)_{k}+\left(\beta \oplus \alpha^{-1}\right)_{k}\right) / \#\left(T^{k}\right) .
$$

The space $\Lambda$ is a perfect compact totally disconnected abelian group.

Let $p$ denote any particular point in $A_{\theta} / \sim$. Then let the map $\psi: \Lambda \rightarrow A_{\theta} / \sim$ be defined by

$$
\psi(\lambda)=\bigcap_{n=0}^{\infty} E_{\theta}^{n}
$$

where each $E_{\theta}^{n}$ is the compouent of $T_{\theta}^{n}$ which contains $\varphi_{2 \pi k}(p)$, where

$$
k=\lambda_{0}+\sum_{j=1}^{n} \lambda_{j} \#\left(T^{j-1}\right) .
$$

By Lemma 1, the map $\psi$ is a bijection. Also, by Lemmas 2 and 3, we see that $\psi$ is a homeomorphism.

By Lemma 7 we see that the map $\bar{\varphi}$ is conjugate to a rotation by $\delta^{0}$ on $\Lambda$, where $\delta^{n}$ is defined by

$$
\left(\delta^{n}\right)_{k}= \begin{cases}1 & \text { if } n=k \\ 0 & \text { otherwise. }\end{cases}
$$

That is, for any $a \in A_{\theta} / \sim$ we have

$$
\bar{\varphi}(a)=\psi\left(\psi^{-1}(a) \oplus \delta^{0}\right) .
$$

If $A$ is solenoidal, then $\oplus \delta^{\prime}$ is an irrational rotation on $\Lambda$.

Note 5. The probability measure $\mu$ on $A_{\theta}$, generated by assigning $\mu$-mass $\left(1 / \#\left(T^{n}\right)\right)$ to each of the \#( $\left.T^{n}\right)=@\left(T^{n}\right)$ components of $T_{\theta}^{n}$, is ergodic and invariant under $\varphi_{2 \pi}$. Unless the components of $A_{\theta}$ are all singleton points, the measure $\mu$ is not defined on all Borel sets in $T_{\theta}$.

Note 6. If $A$ is an orbitally stable attractor of a uniform $C^{0}$ flow $\varphi_{t}$, and class $(A) \in \mathscr{C}(\cdot, P, \cdot)$, then for every point $x \in A$, we have $\omega(x)=A$. If the flow $\varphi_{t}$ is also Lipschitz, then the Hausdorff dimension [Kahane and Salem, 1963] of $(N(x, \varepsilon)$ $\cap A$ ) is independent of choice of $x \in A$ and $\varepsilon>0$. 
Note 7. The Smale horseshoe map [Smale, 1967] on a Smale horseshoe invariant set is conjugate to a shift on a space of doubly infinite sequences. Thus, the Smale horseshoe map on a Smale horseshoe invariant set is not the first return map of any uniform $C^{0}$ flow on the cross-section of an orbitally stable attractor.

We now explicitly calculate the first return map for any invariant circular solenoid $K$ under a uniform $C^{0}$ flow $\varphi_{t}$. We use this result for the standard solenoid $L$ to show some restrictions on the differentiability of $\varphi_{t}$.

Let $\varphi_{t}$ be a uniform $C^{0}$ flow, and let $K$ be an invariant circular solenoid with circular solenoidal maps $\left\{\tau_{k}\right\}_{k=0}^{\infty}$. Let our particular point $p \in K_{\theta}$ be

$$
\sum_{k=0}^{\infty} s_{k}\left(\prod_{j=0}^{k-1} r_{j}\right)
$$

Then the map $\psi: \Lambda \rightarrow K_{\theta}$ is defined by

$$
\psi(\lambda)=\sum_{k=0}^{\infty} s_{k}\left(\prod_{j=0}^{k-1} r_{j}\right) a_{k}(\lambda)
$$

where

$$
a_{k}(\lambda)=\exp \left(\sum_{j=0}^{k} 2 \pi \lambda_{j} i\left(\sum_{n=j}^{k} l_{n}\left(\prod_{s=j}^{n} m_{s}^{-1}\right)\right)\right)
$$

Also, we have

$$
\begin{aligned}
\varphi_{2 \pi}(\psi(\lambda)) & =\sum_{k=0}^{\infty} s_{k}\left(\prod_{j=0}^{k-1} r_{j}\right) a_{k}(\lambda) a_{k}\left(\delta^{0}\right) \\
& =\sum_{k=0}^{\infty} s_{k}\left(\prod_{j=0}^{k-1} r_{j}\right) a_{k}\left(\lambda \oplus \delta^{0}\right) .
\end{aligned}
$$

THEOREM 6. The standard solenoid $L$ is not an invariant set of any uniform $C^{2}$ flow $\varphi_{t}$.

Proof of Theorem 6. For any sequence $\left\{\lambda^{n}\right\}$, such that each $\lambda^{n} \in \Lambda, \lambda^{n} \neq \lambda$, and $\lambda^{n} \rightarrow \lambda$, we have

$$
\lim _{n \rightarrow \infty} \frac{\varphi_{2 \pi}\left(\psi\left(\lambda^{n}\right)\right)-\varphi_{2 \pi}(\psi(\lambda))}{\psi\left(\lambda^{n}\right)-\psi(\lambda)}=\exp \left(2 \pi i \sum_{k=0}^{\infty} 2^{-(k+1)}\right)=1 .
$$

This implies that for any sequence $\left\{z_{n}\right\}$, such that each $z_{n} \in L_{\theta}, z^{n} \neq z$ and $z_{n} \rightarrow z$, we have

$$
\lim _{n \rightarrow \infty} \frac{\varphi_{2 \pi}\left(z_{n}\right)-\varphi_{2 \pi}(z)}{z_{n}-z}=1 \text {. }
$$

We would like to conclude that if $\varphi_{2 \pi}$ is $C^{1}$, then $D \varphi_{2 \pi}(z)=1$ for all $z \in L_{\theta}$. Unfortunately, all we can immediately conclude is that if $\varphi_{2 \pi}$ is analytic, then $D \varphi_{2 \pi}(z)=1$ for all $z \in L_{\theta}$.

By looking at the obvious diagrams, one can show that there exists an $\eta \in(0, \pi / 2)$ such that for any $\varepsilon>0$ and $z \in L_{\theta}$, there exist $z_{1}, z_{2} \in\left(L_{\theta} \backslash\{z\}\right) \cup N(z, \varepsilon)$ such that $\eta<\arg \left(z_{2}-z\right)-\arg \left(z_{1}-z\right)<\pi-\eta$. This allows us to conclude that if $\varphi_{2 \pi}$ is differentiable at $z$, then there are two independent directions where the directional derivative of $\varphi_{2 \pi}$ at $z$ is 1 . Thus if $\varphi_{2 \pi}$ is $C^{1}$, then $D \varphi_{2 \pi}(z)=1$ for all $z \in L_{\theta}$. 
If $\varphi_{2 \pi}$ is $C^{2}$, then by Taylor's theorem,

$$
\varphi_{2 \pi}(z+\Delta z)-\varphi_{2 \pi}(z)-D \varphi_{2 \pi}(z)(\Delta z)=O\left(|\Delta z|^{2}\right) .
$$

Choose $z=\psi(0)$ and $\Delta z_{n}=\psi\left(\delta^{n}\right)-\psi(0)$. Then

$$
\Delta z=(1 / 2) 4^{1-n} \sum_{j=0}^{\infty} 4^{-j}\left(a_{j}\left(\delta^{0}\right)-1\right)
$$

and $\Delta z_{n}$ is of order $4^{-n}$. But

$$
\begin{aligned}
\varphi_{2 \pi}\left(z+\Delta z_{n}\right)-\varphi_{2 \pi}(z)-D \varphi_{2 \pi}(z)\left(\Delta z_{n}\right) \\
=(1 / 2) 4^{1-n} \sum_{j=0}^{\infty} 4^{-j}\left(a_{j}\left(\delta^{0}\right)-1\right)\left(a_{j+n}\left(\delta^{0}\right)-1\right)
\end{aligned}
$$

is of order $8^{-n}$.

Corollary to Theorem 6. The standard solenoid L cannot be imbedded as an orbitally stable attractor of a uniform $C^{2}$ flow.

Note 8. Let $\tau$ be a circular solenoidal map with parameters $r, s, l$ and $m$. Let $K$ denote the circular solenoid each of whose circular solenoidal maps is $\tau$. Using techniques similar to those used in the proof of Theorem 6, one can show that if $K$ is an invariant set of a uniform flow $\varphi_{t}$, then $\varphi_{t}$ cannot be Lipschitz with exponent $p$ for $p>1-(\log m / \log r)$.

\section{Chapter 2. EXISTENCE OF ORBITALly STABlE ATTRACTORS OF UNIFORM $C^{\infty}$ FLOWS}

The primary purpose of this chapter is to prove Theorem 2.

THEOREM 2. For any fixed permissible class there exists a uniform $C^{\infty}$ flow possessing an orbitally stable attractor of that class.

The discussion in $\$ 2.1$ proves our theorem in the case that the fixed permissible class is one of $\mathscr{C}(N, 0,1), \mathscr{C}(N, 1,1), \mathscr{C}(N, 1, M)$, or $\mathscr{C}(N, 1, \omega)$, where $N, M \in \mathbf{Z}^{+}$, $M \geqslant 2$. The corollary to Theorem 7 proves our theorem for the class $\mathscr{C}\left(2^{\omega}, 0,1\right)$, and Theorem 8 proves our theorem for the class $\mathscr{C}\left(2^{\omega}, 1,1\right)$.

We also investigate dynamical and measure-theoretic properties of these attractors. In $\$ 2.4$ we examine the Hausdorff dimensions of our examples of attractors.

2.1 Classes $\mathscr{C}(N, 0,1), \mathscr{C}(N, 1,1), \mathscr{C}(N, 1, M)$ and $\mathscr{C}(N, 1, \omega)$. Let $A \subset T$ be an orbitally stable attractor of a uniform $C^{\infty}$ flow $\varphi_{t}$, and suppose class $(A) \in \mathscr{C}(1, \beta, \gamma)$. Let $\tau$ be a circular solenoid map. Then the $C^{\infty}$ flow $\bar{\varphi}_{t}$ on $\tau(T)$ defined by

$$
\bar{\varphi}_{t}=\tau \circ \varphi_{t / \# \tau(T)} \circ \tau^{-1}
$$

can be extended to all of $T$ as a uniform $C^{\infty}$ flow $\hat{\varphi}_{t}$. The set $\tau(A)$ is an orbitally stable attractor of $\hat{\varphi}_{t}$, and class $(\tau(A))=\mathscr{C}(\# \tau(T), \beta, \gamma)$. Thus we only consider examples of attractors of classes $\mathscr{C}(1,0,1), \mathscr{C}(1,1,1), \mathscr{C}(1,1, M)$ and $\mathscr{C}(1,1, \omega)$ in this section. 
(1) The circle $\{(z, \theta) \in T \mid z=0\}$ can easily be imbedded as an orbitally stable attractor with class $\mathscr{C}(1,0,1)$ of a uniform $C^{\infty}$ flow.

(2) The set $\{(z, \theta) \in T \mid \operatorname{Im}(z)=0,-1 / 2 \leqslant \operatorname{Re}(z) \leqslant 1 / 2\}$ can be imbedded as an orbitally stable attractor with class $\mathscr{C}(1,1,1)$ of a uniform $C^{\infty}$ flow.

There are a variety of attractors of class $\mathscr{C}(1,1, M)$ with very different dynamics. Two examples which illustrate this are:

(3) Irrational rotation on the 2-torus.

(4) The Plykin attractor [Plykin, 1974; Newhouse, 1978; Guckenheimer and Holmes, 1983].

Figure 2 is a sketch of the first return map for a version of the Plykin attractor $P$. The set $P_{\theta}$ has exactly one component. Each component of $C P_{\theta}$ which is bounded away from $\operatorname{Fr}\left(T_{\theta}\right)$ is homeomorphic to the open planar disk. Each component of $C P_{\theta}$ shares $P_{\theta}$ as their common frontier in $T_{\theta}$. In both examples (3) and (4), the complement of the attractor has exactly 2 components. If we modify example (4), so that the third iterate of the map in Figure 2 represents the first return map, then the complement of the attractor has exactly 4 components. Similar attractors can be constructed whose class is $\mathscr{C}(1,0, M)$ for any $M \in \mathbf{Z}^{+}, M \geqslant 2$.

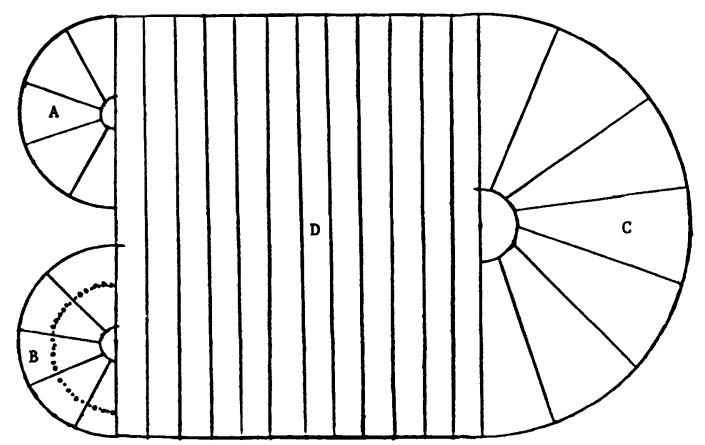

(a) The domain

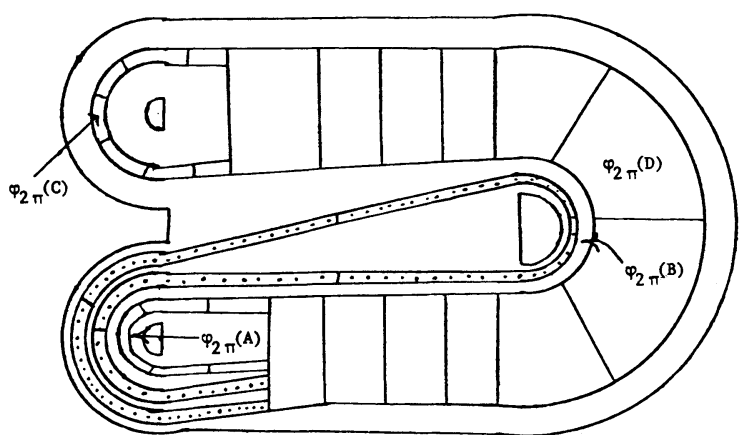

(b) First return map

FIGURE 2. The Plykin map 
We now sketch a modification of the "Lakes of Wada" construction [Yoneyama, 1917; Hocking and Young, 1961]. We construct a compact connected set $W_{\theta} \subset T_{\theta}$ whose complement has exactly three components which we label $B, G$ and $O$, such that

(i) $\operatorname{Fr}(B)=\operatorname{Fr}(G)=W_{\theta}$ holds.

(ii) $\operatorname{Fr}(O)=W_{\theta} \cup \operatorname{Fr}\left(T_{\theta}\right)$ holds.

(iii) The sets $B$ and $G$ are each homeomorphic to the open planar disk.

To preserve the original flavor of the construction, we take the sets $B^{0}, G^{0}$ and $O^{0}$ (Figure 3(a)), to be a lake of blue water, a lake of green water, and an ocean of salt water, respectively. The set $T_{\theta} \backslash\left(B^{0} \cup G^{0} \cup O^{0}\right)$ is taken to be an island. At time $t=1$ we dig three canals, one from each water source, bringing each type of water to within $2^{-1}$ of every point of land (Figure $3(\mathrm{~b})$ ). These larger water sources we label $B^{1}, G^{1}$ and $O^{1}$, respectively. Similarly, for each $n \in \mathbf{Z}^{+}$, we add a canal to each of $B^{n-1}, G^{n-1}$ and $O^{n-1}$, so that each type of water is brought to within $2^{-n}$ of every point of land. We take each of the bodies of water to be open and let $B=\cup B^{n}$, $G=\cup G^{n}$ and $O=\cup O^{n}$ hold. Then $W_{\theta}=T_{\theta} \backslash(B \cup G \cup O)$.

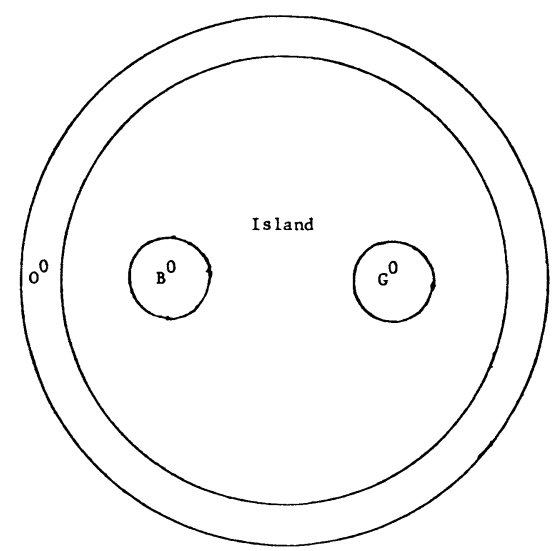

(a) $t=0$

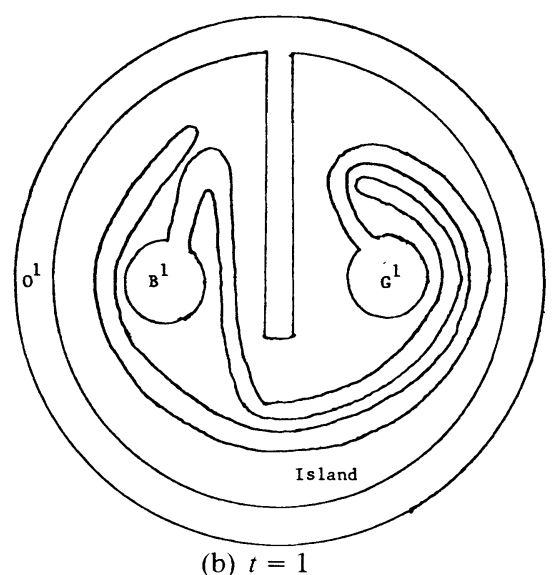

FIGURE 3. Lakes of Wada construction 
By adding another color lake at each time $t \in \mathbf{Z}^{+}$, we can construct a compact connected set $V_{\theta} \subset T_{\theta}$, whose complement has an infinite number of components which we label $\left\{J^{k}\right\}_{k=0}^{\infty}$, such that

(i) $\operatorname{Fr}\left(J^{k}\right)=V_{\theta}$ holds if $k \geqslant 1$.

(ii) $\operatorname{Fr}\left(J^{0}\right)=V_{\theta} \cup \operatorname{Fr}\left(T_{\theta}\right)$.

(iii) The sets $\left\{J^{k}\right\}_{k=1}^{\infty}$ are each homeomorphic to the open planar disk.

Let $V$ denote the set $\left\{(z, \bar{\theta}) \in T \mid z \in V_{\theta}\right\}$. One can imbed $V$ as an orbitally stable attractor of a uniform $C^{\infty}$ flow, and we have class $(V)=\mathscr{C}(1,1, \omega)$.

2.2 Class $\mathscr{C}\left(2^{\omega}, 0,1\right)$ and circular solenoids. Now we consider the problem of imbedding circular solenoids as orbitally stable attractors of uniform $C^{p}$ flows. The corollary to Theorem 6 states that the standard solenoid cannot be imbedded as an orbitally stable attractor of a uniform $C^{2}$ flow. Here we find that if the twists (i.e. $\left.\left|l_{k} / m_{k}\right|\right)$ of the maps $\left\{\tau_{k}\right\}$ decrease quickly enough, then the circular solenoid $K$ of $\left\{\tau_{k}\right\}$ can be imbedded as an orbitally stable attractor of a uniform $C^{p}$ flow for large $p$. More precisely we have

THEOREM 7. Let $\left\{\tau_{k}\right\}_{k=0}^{\infty}$ be a sequence of circular solenoidal maps with corresponding $\left\{r_{k}\right\},\left\{s_{k}\right\},\left\{l_{k}\right\}$ and $\left\{m_{k}\right\}$. Then the circular solenoid $K$ of $\left\{\tau_{k}\right\}$ can be imbedded as an orbitally stable attractor of a uniform $C^{p}$ flow $\varphi_{t}$, where $p$ denotes

$$
\sup \left(\left\{n \in \mathbf{Z}^{+} \mid \sigma(n)<\infty\right\} \cup\{0\}\right),
$$

and

$$
\sigma(n)=\sum_{k=0}^{\infty}\left(\frac{1-\left(r_{k}+s_{k}\right)}{2}\right)^{-n}\left(\prod_{j=0}^{k-1} r_{j}\right)^{1-n} \frac{l_{k}}{m_{k}}\left(b_{k}\right)^{n / 2},
$$

and

$$
b_{k}=\left(1+\left(\sum_{j=0}^{k-1} l_{j}\left(\prod_{s=0}^{j} m_{s}^{-1}\right)\right)^{2}\right) .
$$

Proof of Theorem 7. Let $f: \mathbf{R} \rightarrow[0,1]$ denote a $C^{\infty}$ nonincreasing function such that $f(0)=1$ and $f(1)=0$. Let $f_{k}: \mathbf{R} \rightarrow[0,1]$ be defined by

$$
f_{k}(x)=f\left(\frac{x-\left(1-\varepsilon_{k}\right)}{\varepsilon_{k}}\right),
$$

where $\varepsilon_{k}=\left(1-\left(r_{k}+s_{k}\right)\right) / 2$.

Let $V^{k}$ be the $C^{\infty}$ vector field on $\bar{T}$ defined by (Figure 4(a))

$$
\begin{gathered}
V^{k}=D\left(\tau_{0} \circ \tau_{1} \circ \cdots \circ \tau_{k-1}\right)\left(f_{k}(|z|)(i z)\left(l_{k} / m_{k}\right), 0\right), \quad k \in \mathbf{Z}^{+}, \\
V^{0}=\left(f_{0}(|z|)(i z)\left(l_{0} / m_{0}\right), 0\right),
\end{gathered}
$$

where $D$ is the derivative operator. Then

$$
\begin{gathered}
\left\|V^{0}\right\|_{n} \leqslant\left\|\left(f_{0}(|z|)(i z), 0\right)\right\|_{n}\left(l_{0} / m_{0}\right), \\
\left\|V^{k}\right\|_{n} \leqslant\left\|\left(f_{k}(|z|)(i z), 0\right)\right\|_{n}\left(l_{k} / m_{k}\right)\left(\prod_{j=0}^{k-1} r_{j}\right)^{1-n}\left(b_{k}\right)^{n / 2}, \quad k \in \mathbf{Z}^{+},
\end{gathered}
$$


and

$$
\left\|\left(f_{k}(|z|)(i z), 0\right)\right\|_{n}=\|(f(|z|)(i z), 0)\|_{n}\left(\varepsilon_{k}\right)^{-n},
$$

where $\left\|V^{k}\right\|_{n}$ denotes the supremum over all directions $\hat{u}$ of the $n$th derivative of $V^{k}$ in the direction $\hat{u}$. The factor $\left(\prod_{j=0}^{k-1} r_{j}\right)^{1-n}$ results from a rescaling of the vector field $\left(f_{k}(|z|)(i z)\left(l_{k} / m_{k}\right), 0\right)$. The factor $\left(b_{k}\right)^{n / 2}$ results from the twisting of the map $\left(\tau_{0} \circ \tau_{1} \circ \cdots \circ \tau_{k-1}\right)$.

Let $\varphi_{t}^{*}$ denote the flow of the vector field $(0,1)+\sum_{k=0}^{\infty} V^{k}$. Then $\varphi_{t}^{*}$ is a uniform $C^{p}$ flow. Each $T^{n}$ is invariant under $\varphi_{t}^{*}$, thus $K$ is also.

For $k \in \mathbf{Z}, k \geqslant 0$, let $U_{\theta}^{k}$ be a $C^{\infty}$ vector field on $\bar{T}_{\theta}$ as in Figure 4(b). That is,

(i) $U_{\theta}^{k}$ is symmetric with respect to a rigid rotation of $2 \pi l_{k} / m_{k}$ radians about $z=0$.

(ii) $U_{\theta}^{k}$ is transverse inward to the circles of radius between $1-\varepsilon_{k}$ and 1 centered at $z=0$.

(iii) $U_{\theta}^{k}$ is either 0 or transverse inward to the circles of radius between $r_{k}\left(1-\varepsilon_{k+1}\right)$ and $r_{k}$, centered at the $m_{k}$ points $s_{k} \exp \left(2 \pi l_{k} j i / m_{k}\right)$, where $j \in \mathbf{Z} \bmod \left(m_{k}\right)$.

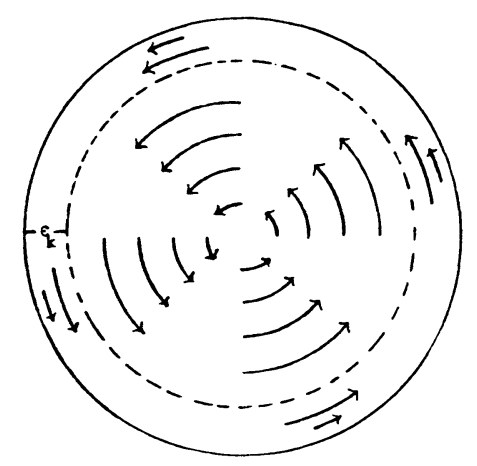

(a) The vector field $\left(f(|z|)(i z)\left(l_{k} / m_{k}\right), 0\right)$ on $\bar{T}_{\theta}$

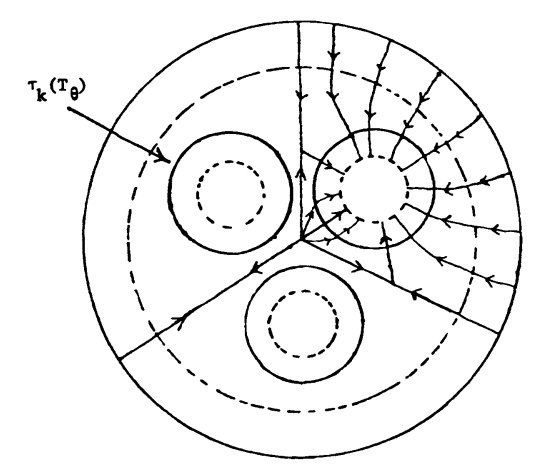

(b) Some flow lines of the vector field $U_{\theta}^{k}$

FIGURE 4. Construction of the vector fields $V^{k}$ and $W^{k}$ for circular solenoid 
(iv) Almost every point in $T_{\theta}$ flows eventually into

$$
\left(\tau_{k}(T)\right)_{\theta}=\bigcup_{j=1}^{m_{k}}\left\{\left|z-s_{k} \exp \left(2 \pi l_{k} j i / m_{k}\right)\right|<r_{k}\right\},
$$

under the flow of $U_{\theta}^{k}$.

(iv) $U_{\theta}^{k}$ and all of $U_{\theta}^{k}$ 's derivatives are 0 on $\{|z|=1\}$.

(vi) $U_{\theta}^{k}$ is 0 on

$$
\bigcup_{j=1}^{m_{k}}\left\{\left|z-s_{k} \exp \left(2 \pi l_{k} j i / m_{k}\right)\right|<r_{k}\left(1-\varepsilon_{k+1}\right)\right\} .
$$

Let the $C^{\infty}$ vector field $W^{k}$ on $\bar{T}$ be defined by

$$
W^{k}=\bigcup_{0<t \leqslant 2 \pi} D \varphi_{t}^{*} U_{\theta}^{k} / \gamma_{k}, \quad k=0
$$

and

$$
W^{k}=\bigcup_{0<t \leqslant 2 \pi \#\left(T^{k}\right)} D \varphi_{t}^{*} D\left(\tau_{0} \circ \tau_{1} \circ \cdots \circ \tau_{k-1}\right) U_{\theta}^{k} / \gamma_{k}, \quad k \geqslant 1,
$$

where $\gamma_{k} \in \mathbf{R}^{+}$is chosen sufficiently large so that for all $0 \leqslant n \leqslant k, n \in \mathbf{Z}$, we have $\left\|W^{k}\right\|_{n}<2^{-k}$.

Let $\varphi_{t}$ denote the flow of the $C^{p}$ vector field

$$
(0,1)+\sum_{k=0}^{\infty}\left(V^{k}+W^{k}\right)
$$

Then $\varphi_{t}$ is a uniform $C^{p}$ flow. Each $T^{n}$ is positively invariant. By construction, every point in the set

$$
\left\{\tau_{0} \circ \tau_{1} \circ \cdots \circ \tau_{n-1}\right\}\left\{(z, \theta) \in T\left|\left(1-\varepsilon_{k}\right)<\right| z \mid<1\right\}
$$

flows eventually into the set

$$
\left\{\tau_{0} \circ \tau_{1} \circ \cdots \circ \tau_{n-1}\right\}\left\{(z, \theta) \in T|| z \mid<\left(1-\varepsilon_{n}\right)\right\}
$$

under $\varphi_{t}$. Also, by construction almost every point in the set

$$
\left\{\tau_{0} \circ \tau_{1} \circ \cdots \circ \tau_{n-1}\right\}\left\{(z, \theta) \in T|| z \mid<\left(1-\varepsilon_{n}\right)\right\}
$$

flows eventually into $T^{n+1}$. So almost every point in $T^{n}$ flows eventually into $T^{n+1}$. Since the flow $\varphi_{t}^{*}$ is measure preserving and the vector field $\sum_{k=0}^{\infty} W^{k}$ is $C^{1}$ (actually $C^{\infty}$ ), the measures induced by $\varphi_{t}$ are absolutely continuous. Thus, by Theorem 4 , the circular solenoid $K$ is an orbitally stable attractor of the uniform $C^{p}$ flow $\varphi_{t}$.

COROllary to TheOREM 7. There exists a uniform $C^{\infty}$ flow possessing a circular solenoid as an orbitally stable attractor of class $\mathscr{C}\left(2^{\omega}, 0,1\right)$.

2.3 Class $\mathscr{C}\left(2^{\omega}, 1,1\right)$. We now use a sequence of maps $\left\{\rho_{k}\right\}_{k=0}^{\infty}$, where each $\rho_{k}$ : $\bar{T} \rightarrow T$ is injective and each $\rho_{k}(T)$ is a uniform open solid torus with winding number $m_{k}$, to construct a solenoid $A$ which can be imbedded as an orbitally stable attractor of a uniform $C^{\infty}$ flow. These maps $\left\{\rho_{k}\right\}$ are used similarly to the circular solenoidal map $\left\{\tau_{k}\right\}$ of Theorem 7 , but

$$
\lim _{n \rightarrow \infty} \operatorname{diam}\left(\left(\rho_{0} \circ \rho_{1} \circ \cdots \circ \rho_{n}\right)\left(T_{\theta}\right)\right)>0
$$

holds. So, $A_{\theta}$ is not a Cantor set. One can even choose the maps $\left\{\rho_{k}\right\}$ so that $\mathscr{M}(A)>0$. 
THEOREM 8. There exist uniform $C^{\infty}$ flows $\varphi_{t}$ and $\varphi_{t}^{\prime}$, possessing orbitally stable attractors $A$ and $A^{\prime}$, respectively, so that class $(A)=$ class $\left(A^{\prime}\right)=\mathscr{C}\left(2^{\omega}, 1,1\right)$, and $\mathscr{M}(A)=0$ and $\mathscr{M}\left(A^{\prime}\right)>0$.

Proof of Theorem 8. Let $f: \mathbf{R} \rightarrow[0,1]$ denote a $C^{\infty}$ nonincreasing function such that $f(0)=1$ and $f(1)=0$. Let $f_{k}: \mathbf{R} \rightarrow[0,1]$ be defined by

$$
f_{k}(x)=f\left(\frac{x-\left(1-\varepsilon_{k}\right)}{\varepsilon_{k}}\right) \text {. }
$$

We will choose three sequences $\left\{\varepsilon_{k}\right\},\left\{\psi_{k}\right\}$ and $\left\{m_{k}\right\}$, where each $\varepsilon_{k} \in(0,1)$, each $\psi_{k}: \bar{T}_{\theta} \rightarrow T_{\theta}$ is $C^{\infty}$ and injective and each $m_{k}=\#\left(T^{k+1}\right) / \#\left(T^{k}\right)$. Our $k$ th noncircular solenoidal map $\rho_{k}: T \rightarrow T$ is defined by

$$
\rho_{k}(z, \theta)=\left(\psi_{k}(z) \exp (i \theta), m_{k} \theta\right) .
$$

Thus $T^{0}=T$ and for $k \in \mathbf{Z}^{+}$, we have

$$
T^{k}=\left(\rho_{0} \circ \rho_{1} \circ \cdots \circ \rho_{k-1}\right)(T) .
$$

And we define

$$
V^{0}=\left(f_{0}(|z|)(i z) / m_{0}, 0\right)
$$

and for $k \in \mathbf{Z}^{+}$,

$$
V^{k}=D\left(\rho_{0} \circ \rho_{1} \circ \cdots \circ \rho_{k-1}\right)\left(f_{k}(|z|)(i z) / m_{k}, 0\right) .
$$

Also, $\varphi_{t}^{*}$ is the flow of the vector field $(0,1)+\sum_{k=0}^{\infty} V^{k}$.

Choose $\varepsilon_{0}=1 / 8$. Given $\left\{\varepsilon_{k}\right\}_{k=0}^{n},\left\{\psi_{k}\right\}_{k=0}^{n}$ and $\left\{m_{k}\right\}_{k=0}^{n}$, we choose $\varepsilon_{n+1}, \psi_{n+1}$ and $m_{n+1}$ as follows (Figure 5(a)):

(i) $m_{n+1}$ is sufficiently large so that $\max \left\{\left\|V^{n}\right\|_{k}\right\}_{k=0}^{n}<2^{-n}$.

(ii) $m_{n+1}>2 \pi / \varepsilon_{n}$.

(iii) $\varepsilon_{n+1} \leqslant 2^{-(n+3)} /\left\|D\left(\rho_{0} \circ \rho_{1} \circ \cdots \circ \rho_{n}\right)\right\|_{1}$.

(iv) $\psi_{n+1}\left(T_{\theta}\right) \subset\left\{|z|<\left(1-\varepsilon_{n+1}\right)\right\}$.

(v) The sets $\left\{\psi_{n+1}\left(\bar{T}_{\theta}\right) \exp \left(2 \pi k i / m_{n+1}\right)\right\}_{k=1}^{m_{n+1}}$ are disjoint.

(vi) $B\left(\psi_{n+1}\left(T_{\theta}\right), \varepsilon_{n+1}\right) \supset\left\{|z|=\left(1-\varepsilon_{n+1}\right)\right\}$.

(vii) $\psi_{n+1}\left(T_{\theta}\right)$ contains no ball of radius $\varepsilon_{n+1}$.

(viii) $\mathscr{M}\left(\psi_{n+1}\left(T_{\theta}\right)\right)<2^{-n} / m_{n+1}\left\|D\left(\rho_{0} \circ \rho_{1} \circ \cdots \circ \rho_{n}\right)\right\|_{1}^{2}$.

By (i) the flow $\varphi_{t}^{*}$ is $C^{\infty}$ and uniform. By (ii) every point of

$$
\left(\rho_{0} \circ \rho_{1} \circ \cdots \circ \rho_{n-1}\right)\left\{|z|=\left(1-\varepsilon_{n}\right)\right\}
$$

flows to within $2^{-(n+2)}$ of every other point, under $\varphi_{t}^{*}$. By (iii) and (vii), $A_{\theta}=\cap T_{\theta}^{n}$. By (iii) and (vi) we have

$$
\operatorname{diam}\left(\left(\rho_{0} \circ \rho_{1} \circ \cdots \circ \rho_{n}\right)\left(T_{\theta}\right)\right)>\operatorname{diam}\left(\left(\rho_{0} \circ \rho_{1} \circ \ldots \circ \rho_{n-1}\right)\left(T_{\theta}\right)\right)-2^{-(n+2)} .
$$

Thus $A_{\theta}$ has some component whose diameter is at least $3 / 2$ and $A_{\theta}$ is not a Cantor set. By (iv) each $T^{n}$ is invariant under $\varphi_{t}^{*}$. By (v) each $T^{n+1}$ is an open solid torus with winding number $\prod_{k=0}^{n} m_{k}$ and $\rho_{n+1}$ is injective. By (viii), $\mathscr{M}\left(T_{\theta}^{n+1}\right)<2^{-n}$. 


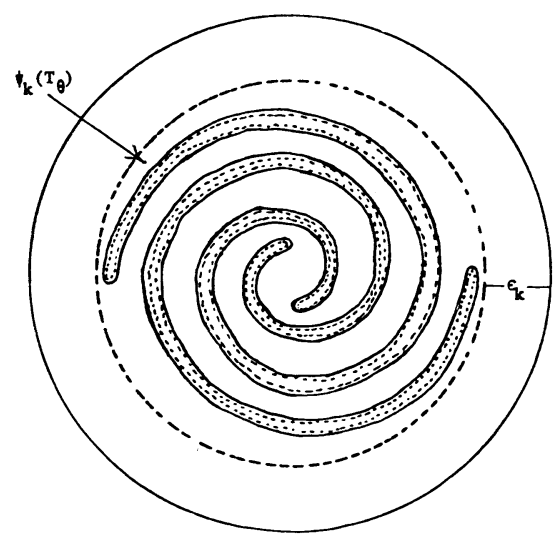

(a) Cross-section of $\rho_{k}(T)$, with $m_{k}=2$

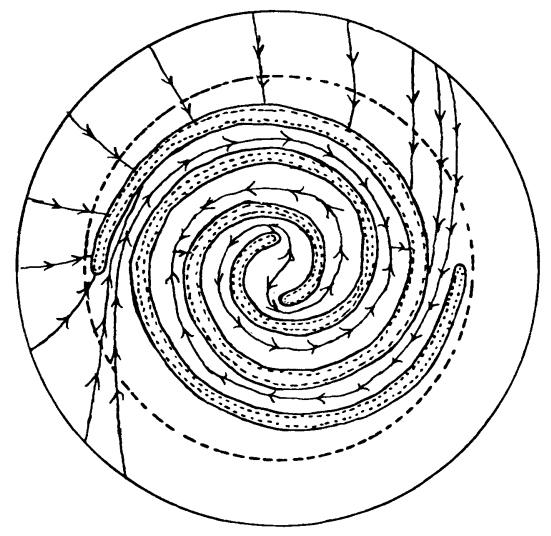

(b) Some flow lines of the vector field $U_{\theta}^{k}$

Figure 5. Construction of an orbitally stable attractor of class $\mathscr{C}\left(2^{\omega}, 1,1\right)$

Let $U_{\theta}^{k}$ be a $C^{\infty}$ vector field on $\bar{T}_{\theta}$ as in Figure 5(b). That is,

(i') $U_{\theta}^{k}$ is symmetric with respect to a rigid rotation of $2 \pi\left(m_{k}\right)^{-1}$ radians about $z=0$.

(ii') $U_{\theta}^{k}$ is transverse inward to the circles of radius between $1-\varepsilon_{k}^{\prime}$ and 1 centered at $z=0$.

(iii') $U_{\theta}^{k}$ is either 0 or transverse inward to the image under $\psi_{k+1}$ of the circles of radius between $1-\varepsilon_{k+1}$ and 1 centered at $z=0$.

(iv') Almost every point in $T_{\theta}$ flows eventually into

$$
\left(\rho_{k+1}(T)\right)_{\theta}=\bigcup_{j=1}^{m_{k+1}} \psi_{n+1}\left(T_{\theta}\right) \exp \left(2 \pi j i / m_{k+1}\right)
$$

under the flow of $U_{\theta}^{k}$.

$\left(\mathrm{v}^{\prime}\right) U_{\theta}^{k}$ and all of $U_{\theta}^{k}$ 's derivatives are 0 on $\{|z|=1\}$.

(vi') $U_{\theta}^{k}$ is 0 on $\left(\rho_{k+1}(T)\right)_{\theta}$.

Let the $C^{\infty}$ vector field $W^{k}$ on $\bar{T}$ be defined by

$$
W^{k}=\bigcup_{0<t \leqslant 2 \pi} U_{\theta}^{k} / \gamma_{k} \quad \text { for } k=0,
$$


and

$$
W^{k}=\bigcup_{0<t \leqslant 2 \pi \#\left(T^{k}\right)} D \varphi_{t}^{*} D\left(\rho_{0} \circ \rho_{1} \circ \cdots \circ \rho_{k-1}\right) U_{\theta}^{k} / \gamma_{k} \quad \text { for } k \in \mathbf{Z}^{+},
$$

where $\gamma_{k} \in \mathbf{R}^{+}$is chosen sufficiently large so that for all $n \in[0, k], n \in \mathbf{Z}$, we have $\left\|W^{k}\right\|_{n}<2^{-k}$, and also for any fixed $k \in \mathbf{Z}^{+}$, every point in the set

$$
\left(\rho_{0} \circ \rho_{1} \circ \cdots \circ \rho_{k-1}\right)\left\{(z, \theta) \in T|| z \mid=\left(1-\varepsilon_{k}\right)\right\}
$$

flows to within $2^{-(k+1)}$ of every other such point, under the flow of

$$
(0,1)+W^{k}+\sum_{n=0}^{\infty} V^{n}
$$

Let $S^{n}$ be the uniform open solid torus

$$
\left\{(z, \theta) \in T|| z \mid<\left(1-\varepsilon_{n}\right)\right\} \text { for } n=0,
$$

and

$$
\left(\rho_{0} \circ \rho_{1} \circ \cdots \circ \rho_{n-1}\right)\left\{(z, \theta) \in T|| z \mid<\left(1-\varepsilon_{n}\right)\right\} \text { for } n \in \mathbf{Z}^{+} .
$$

Let $\varphi_{t}$ denote the flow of

$$
(0,1)+\sum_{n=0}^{\infty}\left(V^{n}+W^{n}\right)
$$

Then $\varphi_{t}$ is a uniform $C^{\infty}$ flow, and $\left\{S^{n}\right\}_{n=0}^{\infty}$ is a positively invariant solenoidal $T$-sequence for $A$. For each $n \geqslant 0$, almost every point in $S^{n}$ flows eventually into $S^{n+1}$, also every point in $\operatorname{Fr}\left(S^{n}\right)$ flows to within $2^{-(n+1)}$ of every other point in $\operatorname{Fr}\left(S^{n}\right)$. Thus, by Theorem 4 , the set $A$ is an orbitally stable attractor of class $\mathscr{C}\left(2^{\omega}, 1,1\right)$ for the uniform $C^{\infty}$ flow $\varphi_{t}$.

The construction of $\varphi_{t}^{\prime}$ and $A^{\prime}$ is the same as that of $\varphi_{t}$ and $A$, but with

(iii') $\varepsilon_{n+1} \leqslant 2^{-(n+3)} /\left(\left\|D\left(\rho_{0} \circ \rho_{1} \circ \cdots \circ \rho_{n}\right)\right\|_{1}\right)^{2}$, and

(viii') $\left(1-m_{n+1} \mathscr{M}\left(\psi_{n+1}\left(T_{\theta}\right)\right)\right)<2^{-(n+2)} /\left(\left\|D\left(\rho_{0} \circ \rho_{1} \circ \ldots \circ \rho_{n}\right)\right\|_{1}\right)$, in place of (iii) and (viii), respectively. In this case we have

$$
\mathscr{M}\left(\left(\rho_{0} \circ \rho_{1} \circ \cdots \circ \rho_{n}\right)(T)\right)_{\theta}>\mathscr{M}\left(\left(\rho_{0} \circ \rho_{1} \circ \cdots \circ \rho_{n-1}\right)(T)\right)_{\theta}-2^{-(n+2)} .
$$

So $\mathscr{M}\left(A_{\theta}\right)>1 / 2$ and $\mathscr{M}(A)>0$.

2.4 Hausdorff dimension. Let $f: T \rightarrow T$ be a Lipschitz map, such that $f^{-1}$ exists and is also Lipschitz. Then for any set $K \subset T$ we have $\operatorname{HD}(K)=\operatorname{HD}(f(K))$; cf. [Kahane and Salem, 1963].

Let $A$ be an orbitally stable attractor of a uniform Lipschitz flow. Then we have $\operatorname{HD}(A)=1+\operatorname{HD}\left(A_{\theta}\right)$ and $1 \leqslant \mathrm{HD}(A) \leqslant 3$.

For any class $\mathscr{C}(\alpha, 1, \gamma)$, where $\alpha \in \mathbf{Z}^{+}$and $\gamma \in \mathbf{Z}^{+} \cup\{\omega\}$, and any $\delta \in[2,3]$, one can use a modification of the "Lakes of Wada" construction in which one carefully controls the widths of the canals, to construct an orbitally stable attractor of a uniform $C^{\infty}$ flow whose class is $\mathscr{C}(\alpha, 1, \gamma)$ and whose Hausdorff dimension is $\delta$. Similarly one can construct an orbitally stable attractor whose local Hausdorff dimension varies over the attractor. 
Definition 12. The local Hausdorff dimension of a nonempty set $A \subset T$ at a point $x \in A$ is denoted by $\operatorname{HD}_{l}(A, x)$, and defined by

$$
\mathrm{HD}_{l}(A, x)=\lim _{\varepsilon \downarrow 0} \mathrm{HD}(A \cap N(x, \varepsilon)) \text {. }
$$

If $A$ is an orbitally stable attractor of a uniformly Lipschitz flow, and class $(A) \in\left\{\mathscr{C}(\alpha, 0,1) \mid \alpha \in \mathbf{Z}^{+} \cup\left\{2^{\omega}\right\}\right\}$, then it follows from Note 6 that the local Hausdorff dimension of $A$ is constant over $A$.

THEOREM 9. For each fixed $\delta \in[1,3]$, there exists a uniform $C^{\infty}$ flow, possessing an orbitally stable attractor having Hausdorff dimension $\delta$.

Proof of Theorem 9. By the preceding discussion it suffices to prove the following lemma.

Lemma 8. For each fixed $\delta \in[1,2]$, there exists a uniform $C^{\infty}$ flow $\varphi_{t}$, possessing an orbitally stable circular solenoidal attractor $K$, having Hausdorff dimension $\delta$.

Question. Does there exist any uniform Lipschitz flow possessing an orbitally stable attractor of class $\mathscr{C}\left(2^{\omega}, 0,1\right)$, having Hausdorff dimension greater than 2 ?

Proof of LemMA 8. For $\delta \in(1,2]$ choose a sequence of circular solenoidal maps $\left\{\tau_{k}\right\}$ so that for all $k \geqslant 0$

(i) $m_{k}>6$

(ii) $r_{k}=\left(m_{k}\right)^{\gamma}$,

(iii) $\sigma(k)<\infty$, and

(iv) $S_{k}=1 / 2$,

where $\gamma=1 /(1-\delta)$.

Each of the $\prod_{k=0}^{n-1} m_{k}$ components of $T_{\theta}^{n}$ is a 2-disk with radius $\prod_{k=0}^{n-1}\left(m_{k}\right)^{\gamma}$ and is at least a distance $\Pi_{k=0}^{n-1}\left(m_{k}\right)^{\gamma}$ away from any other component of $T_{\theta}^{n}$.

For any $n \geqslant 1$, we can cover $T_{\theta}^{n}$ with $\prod_{k=0}^{n-1} m_{k} 2$-disks of radius $\prod_{k=0}^{n-1}\left(m_{k}\right)^{\gamma}$, so the Hausdorff dimension of $K_{\theta}$ is bounded above by

$$
\lim _{n \rightarrow \infty} \frac{-\log \prod_{k=0}^{n-1} m_{k}}{\log \prod_{k=0}^{n-1}\left(m_{k}\right)^{\gamma}}=\delta-1 .
$$

To show that the Hausdorff dimension of $K_{\theta}$ is at least $(\delta-1)$, it suffices to show that the probability measure $\mu$ of Note 5 satisfies the Hölder condition $\mu(B(x, \varepsilon)) \leqslant$ $\varepsilon^{(\delta-1)}$. It suffices to prove this for

$$
\prod_{k=0}^{n}\left(m_{k}\right)^{\gamma} \leqslant \varepsilon \leqslant \prod_{k=0}^{n-1}\left(m_{k}\right)^{\gamma}
$$

Case I.

$$
(1 / 6) \prod_{k=0}^{n-1}\left(m_{k}\right)^{\gamma} \leqslant \varepsilon \leqslant \prod_{k=0}^{n-1}\left(m_{k}\right)^{\gamma} \text {. }
$$

Since every component of $T_{\theta}^{n}$ is at least a distance $\prod_{k=0}^{n-1}\left(m_{k}\right)^{\gamma}$ away from any other component, we have

$$
\mu(B(x, \varepsilon)) \leqslant \prod_{k=0}^{n-1}\left(m_{k}\right)^{-1} \leqslant 6^{(\delta-1)} \varepsilon^{(\delta-1)} \leqslant 6 \varepsilon^{(\delta-1)} .
$$


Case II.

$$
\prod_{k=0}^{n}\left(m_{k}\right)^{\gamma} \leqslant \varepsilon \leqslant(1 / 6) \prod_{k=0}^{n-1}\left(m_{k}\right)^{\gamma} .
$$

Let $\rho$ denote $3 \varepsilon /(2 \pi)\left(\prod_{k=0}^{n-1}\left(m_{k}\right)^{\gamma}\right)$ or, approximately the maximum fraction of a circle of radius $(1 / 3) \prod_{k=0}^{n-1}\left(m_{k}\right)^{\gamma}$ that a disk of radius $\varepsilon$ can cover. Clearly $2 \rho$ is greater than that maximum fraction. The components of $T_{\theta}^{n+1}$ are spaced evenly about circles of radius $(1 / 2) \prod_{k=0}^{n}\left(m_{k}\right)^{\gamma}$ centered at the centers of each component of $T_{\theta}^{n}$ (Figure 6). So, we have

$$
\begin{aligned}
\mu(B(x, \varepsilon)) & \leqslant 2 \rho \prod_{k=0}^{n-1}\left(m_{k}\right)^{-1}+2 \prod_{k=0}^{n}\left(m_{k}\right)^{-1} \\
& \leqslant(3 / \pi) \varepsilon \prod_{k=0}^{n-1}\left(m_{k}\right)^{(\delta-2) \gamma}+2 \varepsilon^{(\delta-1)} \\
& \leqslant(3 / \pi)(\varepsilon)(6 \varepsilon)^{(\delta-2)}+2 \varepsilon^{(\delta-1)}<b \varepsilon^{(\delta-1)} .
\end{aligned}
$$

For $\delta=1$, we choose a sequence of circular solenoidal maps $\left\{\tau_{k}\right\}$ so that we have $\sigma(p)<\infty$ for all $p \in \mathbf{Z}^{+}$and

$$
\lim _{k \rightarrow \infty} \frac{-\log m_{k}}{\log r_{k}}=0
$$

Then we have

$$
\lim _{n \rightarrow \infty} \frac{-\log \prod_{k=0}^{n} m_{k}}{\log \prod_{k=0}^{n} r_{k}}=0,
$$

and the Hausdorff dimension of $K_{\theta}$ is 0 .

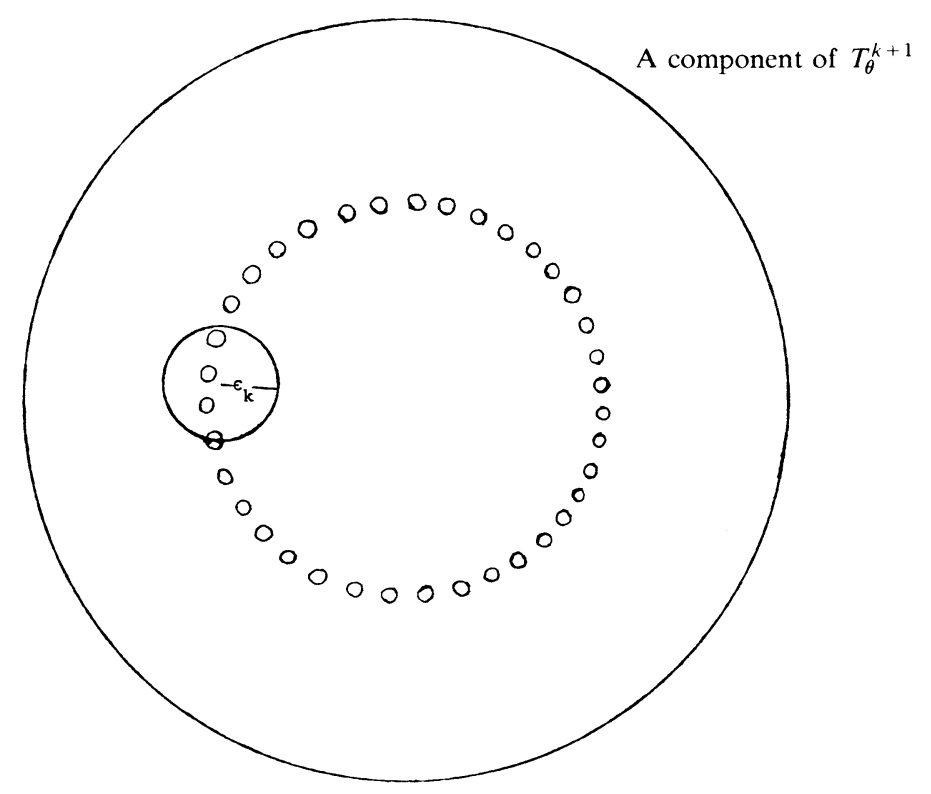

FIGURE 6. One component of $T_{\theta}^{k}$ 


\section{BIBLIOGRAPHY}

R. Bowen [1978], On Axiom A diffeomorphisms, CBMS Regional Conf. Ser. Math., no. 35, Amer. Math. Soc., Providence, R.I.

R. Bowen and J. Franks [1976], The periodic points of maps of the disk and the interval, Topology 15, pp. 337-342.

G. Duffing [1918], Erzwungene Schwingungen bei Veränderlicher Eigenfrequenz, Braunschweig.

C. H. Edwards [1962], Concentric solid tori in the 3-sphere, Trans. Amer. Math. Soc. 102, pp. 1-17.

J. Guckenheimer and P. Holmes [1983], Nonlinear oscillations, dynamical systems, and bifurcations of vector fields, Appl. Math. Sci., vol. 42, Springer-Verlag, New York.

J. G. Hocking and G. S. Young [1961], Topology, Addison-Wesley, Reading, Mass., p. 143.

J. P. Kahane and R. Salem [1963], Ensembles parfaits et séries trigonometriques, Actualités Sci. Indust., no. 1301, Hermann, Paris.

S. E. Newhouse [1980], Lectures on dynamical systems, Dynamical Systems, C.I.M.E. Lectures Bressanone, Italy, June 1978, pp. 209-312.

R. Plykin [1974], Sources andd sinks of A-diffeomorphisms of surfaces, USSR Math.-Sb. 23, pp. 233-253.

S. Smale [1967], Differentiable dynamical systems, Bull. Amer. Math. Soc. 73, pp. 747-817.

K. Yoneyama [1917], Theory of continuous sets of points, Tôhoku Math. J. 12, pp. 43-158.

DePartment of Mathematics, University of Illinois, Urbana, Illinois 61801

Current address: School of Mathematics, Institute for Advanced Study, Princeton, NJ 08540 